\title{
Zero drift regions and control strategies to keep satellite in formation around triangular libration point in the restricted Sun-Earth-Moon scenario
}

\author{
F.J.T. Salazar ${ }^{\mathrm{a}, *}$, O.C. Winter ${ }^{\text {a }}$, E.E. Macau ${ }^{\mathrm{b}}$, J.J. Masdemont ${ }^{\mathrm{c}}$, G. Gómez ${ }^{\mathrm{d}}$ \\ ${ }^{a}$ UNESP-Grupo de Dinâmica Orbital e Planetologia, Guaratinguetá SP 12516-410, Brazil \\ ${ }^{\mathrm{b}}$ Instituto Nacional de Pesquisas Espaciais, Av. dos Astronautas 1758, São José dos Campos SP 12227-010, Brazil \\ ${ }^{\mathrm{c}}$ Departament de Matemàtica Aplicada I, ETSEIB-UPC, Avda. Diagonal 647, 08028 Barcelona, Spain \\ ${ }^{\mathrm{d}}$ Departament de Matemàtica Aplicada i Anàlisi, UB, Gran Vía 585, 08007 Barcelona, Spain
}

Received 1 April 2015; received in revised form 16 June 2015; accepted 1 July 2015

Available online 6 July 2015

\begin{abstract}
In this work, we are interested in avoiding large variations in the mutual distances among multiple satellites and also in controlling their geometric configuration around an Earth-Moon triangular point. Previous studies about triangular libration points have determined the existence of zero drift regions with respect to the nominal trajectory, in which the expansion or contraction of the formation never take place. Our goal is to carry out two different control strategies for a formation near a given nominal trajectory around $L_{4}$ : a bang-off-bang control and a minimum weighted total $\Delta V$ consumption. A linearization relative to the reference trajectory around the triangular libration point is carried out, and different geometrical possibilities in the zero drift regions are studied. To investigate the influence of the gravitational force of the Sun, the BiCircular Four Body Problem is considered here. According to the results obtained, some meaningful insights to allow a proper design of the geometric configuration of the formation are drawn.
\end{abstract}

(C) 2015 COSPAR. Published by Elsevier Ltd. All rights reserved.

Keywords: Formation flying; Zero drift region; Sun-Earth-Moon system; BiCircular Four Body Problem; Equilateral point; Minimum fuel consumption

\section{Introduction}

Satellite formation flying (SFF) means to have two or more spacecraft in orbit such that their relative positions remain constant or obeying a desired certain dynamical configuration along the trajectory (Sholomitsky et al., 1977; Battrick, 2000; Bristow et al., 2000; Burns et al., 2000; Ticker and Azzolini, 2000; Fridlund and Capaccioni, 2002). This concept requires the control over the coordinated motion of a group of satellites, aiming to

\footnotetext{
* Corresponding author. Tel.: +55 12981015572.

E-mail addresses: e7940@hotmail.com (F.J.T. Salazar), ocwinter@ gmail.com (O.C. Winter), elbert.macau@inpe.br(E.E. Macau), josep@ barquins.upc.edu (J.J. Masdemont), gerard@maia.ub.es (G. Gómez).
}

maintain a specific geometric space configuration among the elements of the formation (Sabol et al., 2001). It allows that a group of small satellites, arranged in a space formation flying, operate like a large 'virtual satellite'. For particular applications, this formation will have many benefits over the use of a large single satellite, including reduction of launch, maintenance costs and an unprecedented high resolution (Adams et al., 1996; Folta et al., 1996; Guinn and Boain, 1996; How et al., 1998; Kapila et al., 2000).

Over the past decades, numerous formation flying missions have been conceived. In regard to the planetary orbit scenario, an example of formation is the Landsat 7/Earth Observing-1 pair, a mission designed to enable the development of future Earth imaging observatories that will have a significant increase in performance while reducing cost and 
mass (Flick, 2012). For outer space scenario, there exists interest of major space agencies to position SFF in halo orbits around Lagrangian points $L_{1}$ and $L_{2}$ (Farquhar, 1968; Gómez et al., 2001a; Howell and Marchand, 2005; Marchand and Howell, 2005; Héritier and Howell, 2014) or $L_{4}$ and $L_{5}$ (Simó et al., 1987). An interesting example of this kind of formation flying mission is the New Worlds Observer (NWO) (Cash et al., 2009). NWO consists of a large telescope and an occulter spacecraft in tandem at about 50,000 km apart. The two spacecraft would be flying about the Earth-Sun $L_{2}$ Lagrangian point or in a drift-away solar orbit. Its purpose is to discover and analyze terrestrial extra-solar planets. The NWO planned launch date is about 2018. In the case of Earth-Moon system, $L_{4}$ and $L_{5}$ points could be excellent locations to place space telescopes for astronomical observations or a space station (Schutz, 1977). In addition, Defilippi (1977) made a review of the ideas of American physicist O'Neill (1974) about building space colonies at the $L_{4}$ and $L_{5}$ positions. These space stations could be used as a way-point for traveling to and from the region between Earth's atmosphere and the Moon (cis-lunar space). Despite this advantage, today there are no missions orbiting $L_{4}$ or $L_{5}$ points for any celestial pair of primaries.

One of the main problems of positioning satellites in formation flying is the cost involved in maintaining the formation. This is so because keeping a formation from drifting apart and achieving mission requirements is expected to require significantly more fuel than station keeping a single spacecraft. In the multi-body regime, numerous references in the literature regarding optimal control techniques on formation flight at collinear and triangular libration points can be encountered. Many of these references assume that the radius of the formation (largest separation between the spacecrafts) is no greater than a few kilometers. Hence, the linear approach about a nominal trajectory may give the relevant information about the local dynamics, while classical control theory (e.g. Linear Quadratic Regulator controller) can then be applied to the linearized system (Farquhar, 1971; Hoffman, 1993; Folta et al., 2000; Hamilton, 2001). Dynamical systems approaches are other formation control studies that have been investigated. The goal of these works was the determination of the natural formation dynamics on the center manifold near the libration points (Barden and Howell, 1998a,b, 1999; Howell and Barden, 1999, 2005). In the case of non-natural formations, ${ }^{1}$ Howell and Marchand (2005) used continuous and discrete control strategies to maintain the formations near the $L_{1}$ and $L_{2}$ libration points in the SunEarth/Moon system. Similarly, Marchand and Howell (2005) provided a decentralized control strategy based on existing linear and nonlinear techniques.

Recently, a linear analysis carried out by Gómez et al. (2006), derived via variational equations and using a

\footnotetext{
${ }^{1}$ This type of motion does not exist naturally near libration points.
}

numerical approach, regions of zero relative radial acceleration with respect to a halo orbit near the $L_{2}$ Sun-Earth libration point, that avoid large variations of the mutual distances among the spacecraft. In addition, vehicle-to-vehicle separation was considered small. Analytically, these regions are represented by cone surfaces. The motion on the zero relative radial acceleration cones ideally prevents expansions or contractions of the formation. Gómez et al. (2006) employ discrete control strategies to maintain the natural formation within the zero drift regions. However, it could be that at specific locations along a certain nominal trajectory, the relative acceleration between the two spacecraft never precisely reaches zero. In this sense, Héritier and Howell (2014) introduced the computation of the low drift regions, which correspond to the relative positions that maintain a desired maximum relative acceleration.

Previous studies like those by Catlin and McLaughlin (2007), Wong (2009) and Salazar et al. (2014) on SFF about $L_{4}$ in the Earth-Moon system have been carried out. The motion of formation flying near triangular libration points was studied adopting the Circular Restricted Three Body Problem (CRTBP) model. Catlin and McLaughlin (2007) showed that formations are possible at the triangular points for uncontrolled trajectories due to the stability of stationary solutions. On the other hand, Wong (2009) established that a system control is required and he developed strategies for keeping a spacecraft formation system at $L_{4}$. Salazar et al. (2014) determined analytically and numerically the zero drift regions with respect to a family of periodic orbits about $L_{4}$. Catlin and McLaughlin (2007), Wong (2009) and Salazar et al. (2014) showed that velocity change requirements demanded by control methods would be very small in the CRTBP scenario. Thus, these studies concluded that nonlinear aspects as well as perturbation forces (e.g. solar gravity, solar radiation pressure) are necessary to provide a more real-world accurate descriptions of formation dynamics in flying around equilateral libration points in the Earth-Moon system.

In order to cope with the Sun's perturbation, the SunEarth-Moon bicircular scenario is considered in this work. This dynamical model is a simplified version of the Restricted Four Body Problem, that captures in some sense the basic dynamics of a real four body problem (Simó et al., 1995). The equations of motion of the BiCircular Four Body Problem (BCFBP) in the synodic system can be written in an autonomous fashion; however, $L_{4}$ and $L_{5}$ are no longer equilibrium points, only retaining their geometrical meaning. Using different approaches, Kolenkiewicz and Carpenter $(1967,1968)$ and Gómez et al. (2001b) obtained three periodic orbits in the synodical coordinate frame, that have the same period as the Sun. Two of them are linearly stable, lying far away from the triangular libration points of the CRTBP, while the other one is small and slightly unstable. On the other hand, Simó et al. (1995) and Castellà and Jorba (2000) started on these 
three periodic orbits and obtained a Cantor family of quasi-periodic solutions $(2-D$ tori) for each periodic orbit, which is continued in the vertical ( $z$ and $\dot{z}$ ) direction up to a high distance. Similarly, for moderate vertical amplitudes, the tori on these families have the same stability as the three periodic orbits mentioned above. It is important to mention that stable regions about triangular points seem to persist in the real Earth-Moon system (Jorba, 2000; Hou and Liu, 2010).

The goal of this work is to investigate the existence of stable regions for small formation flight dynamics (maximum distance between the satellites no greater than a few kilometers at most) near bounded solutions ${ }^{2}$ around the equilateral equilibrium point $L_{4}$ of the CRTBP adopting the BCFBP scenario (the same results will hold for $L_{5}$ due to the symmetries of this model). Compared to the CRTBP, the gravitational perturbation from the Sun could approximate better the dynamics near triangular libration points. Furthermore, the method based on the linearization of the relative equations of motion with respect to trajectories around $L_{4}$ is employed. Finally, two discrete control strategies are implemented to maintain a formation on the zero radial acceleration cones: the bang-off-bang and minimum weighted total $\Delta V$ consumption. For both strategies several parametric studies are performed, considering different geometrical possibilities, such as parallel and non-parallel translations between the cone surfaces.

The remainder of this paper is organized as follows. Section 2 describes the equations of motion of the BCFBP, and stable quasi-periodic solutions about $L_{4}$, along which we will assume that the formation is moving. Section 3 defines the regions of zero relative radial acceleration component with respect to a nominal trajectory. Sections 4 and 5 determine the surfaces of zero drift for the quasi-periodic solution, and compute the cost of keeping the geometrical configuration of the spacecraft placed on those regions, using the two discrete control strategies mentioned above. At last, the conclusions are drawn in Section 6.

\section{Stable quasi-periodic orbits around triangular libration point in the BCFBP}

\subsection{The Equations of the $B C F B P$}

Taking into account the Sun's gravitational force in the Earth-Moon system, the BCFBP is formulated under the following assumptions: (i) the movements of the Sun, Earth and Moon take place in the same plane; (ii) the Earth and Moon move around their barycenter in a circular orbit; (iii) the Sun and Earth-Moon barycenter move around their common center in circular orbits. This dynamical model is a modified version of the EarthMoon CRTBP and catches the basic dynamics of the real four-body problem.

\footnotetext{
${ }^{2}$ A solution is called bounded if it is confined to a compact set.
}

Let $\mu$ be the mass of the Moon, $1-\mu$ the mass of the Earth and $\mu_{S}$ the mass of the Sun. Taking as unity the distance between the Earth and the Moon, let $a_{S}$ denote the distance from the Earth-Moon barycenter to the Sun. The equations of motion in the Earth-Moon synodic system, centered at the Earth-Moon barycenter are given by Gómez et al. (2001b)

$$
\begin{aligned}
\ddot{x}-2 \dot{y} & =\Omega_{x}, \\
\ddot{y}+2 \dot{x} & =\Omega_{y}, \\
\ddot{z}+\dot{z} & =\Omega_{z}, \\
\dot{\theta_{S}} & =\omega_{S},
\end{aligned}
$$

where $\omega_{S}$ is the angular speed of the Sun in the synodical system, $\theta_{S}$ is the phase angle of the Sun relative to the Earth-Moon line, $\Omega$ is the perturbed pseudo potential caused by the Sun, and the symbol $\Omega_{i}$ stands for the partial derivatives $\partial \Omega / \partial i$ for $i=x, y, z$.

$$
\begin{aligned}
\Omega= & \frac{1}{2}\left(x^{2}+y^{2}+z^{2}\right)+\frac{1-\mu}{r_{1}}+\frac{\mu}{r_{2}}+\frac{1}{2} \mu(1-\mu) \\
& +\mu_{S}\left(\frac{1}{r_{S}}-\frac{1}{a_{S}^{2}}\left(x \cos \theta_{S}-y \sin \theta_{S}\right)\right),
\end{aligned}
$$

with

$$
\begin{aligned}
& r_{1}=\sqrt{(x+\mu)^{2}+y^{2}+z^{2}}, \\
& r_{2}=\sqrt{(x-1+\mu)^{2}+y^{2}+z^{2}}, \\
& r_{S}=\sqrt{\left(x-a_{S} \cos \theta_{S}\right)^{2}+\left(y+a_{S} \sin \theta_{S}\right)^{2}+z^{2}} .
\end{aligned}
$$

The positions of the Earth, the Moon, and the Sun in the synodical system are $(-\mu, 0,0),(1-\mu, 0,0)$, $\left(a_{S} \cos \theta_{S},-a_{S} \sin \theta_{S}, 0\right)$, respectively. Additionally, even though the system of differential equations (1) is autonomous, $L_{4}$ and $L_{5}$ are no longer equilibrium points, keeping only a geometrical meaning.

\subsection{Solutions around triangular libration point in the $B C F B P$}

In the Sun-Earth-Moon BiCircular Four Body Problem, we take the mass parameter $\mu=1.215058560962404 \times 10^{-2}$, the mass of the Sun $\mu_{S}=328,900.55$, the distance between the Sun and the Earth-Moon barycenter $a_{S}=388.8111430233514$ adim, and the angular speed of the Sun $\omega_{S}=$ 0.92519598551828964 . These values are consistent with the ones used by Gómez et al. (2001b), that applied a continuation method to pass from the Earth-Moon CRTBP to the Sun-Earth-Moon BCFBP and found three periodic orbits with initial phase angle $\theta_{S}=0$, i.e., the initial angle between the Sun-Earth and Earth-Moon lines is equal to zero, and whose periods are equal to the period of revolution of the Sun in the Earth-Moon synodical system: 6.791 units of dimensionless time (about 29 days). Those orbits, denoted by PO1, PO2, and PO3, are shown in Fig. 1 in 
the Earth-Moon rotating frame centered in the EarthMoon barycenter. The system of differential equations (1) were integrated numerically considering the initial states given by Gómez et al. (2001b) and using the BulirschStoer algorithm (Stoer and Bulirsch, 1980; Press et al., 1992), programmed in the $\mathrm{C}$ language, choosing a dimensionless step size $h=1.0 \times 10^{-3}$ and setting a local truncation error of $1.0 \times 10^{-9}$.

The periodic orbits $\mathrm{PO} 2$ and $\mathrm{PO} 3$ are stable solutions about $L_{4}$, i.e., the norm of the eigenvalues of the monodromy matrix associated with the two trajectories are less than one. Those orbits are very large, about 0.6 adim $(230,000 \mathrm{~km})$ along the $x$-axis and $0.4 \operatorname{adim}(153,000 \mathrm{~km})$ along the $y$-axis. Orbit PO1 is an unstable periodic solution about $L_{4}$. This orbit remains within $0.02 \operatorname{adim}(7,600 \mathrm{~km})$ of the libration point. Hence, it 'replaces' the triangular point.

The effects of the initial configuration of the Sun-EarthMoon were studied by Tapley and Lewallen (1964) and Wolaver (1965), and more recently, by Munoz (2008). The problem is to determine the initial phase angle $\theta_{S}$, i.e., the initial position of the Sun, that minimizes the maximum displacement from $L_{4}$. Wolaver (1965), using the approximation of the BCFBP and linearizing the equations of motion in the vicinity of $L_{4}$, obtained three initial positions of the Sun that showed a maximum displacement of $5,349 \mathrm{~km}$ from $L_{4}$ for 400 days. However, this solution does not persist in a more realistic ephemeris-based model. On the other hand, Tapley and Lewallen (1964), including also the inclination of the Sun's orbit in the Sun-Earth-Moon system, found that the motion of the spacecraft initially at $L_{4}$ is very dependent on the initial position of the Sun and that the amplitude of motion about $L_{4}$ and $L_{5}$ is greater than what was predicted by the linearized equations. Similarly, Munoz (2008) obtained an initial value of $\theta_{S}$ in the Sun-Earth-Moon BCFBP, so that, a spacecraft initially at rest at $L_{4}$ remained within $30,000 \mathrm{~km}$ of $L_{4}$ for a propagation time of 7,000 days. However, the resulting trajectory was extremely sensitive to the initial value of $\theta_{S}$.
Therefore, using more realistic model of the Sun-EarthMoon system, Munoz (2008) studied the motion of a spacecraft near the triangular libration points in the Earth-Moon system using the SPICE ephemerides, and found in the year 2007, 12 and 13 epochs in the $L_{4}$ and $L_{5}$ cases, respectively, such that the spacecraft would remain in a close vicinity about triangular points for at least 3,000 days. But in this model again, the resultant motion was sensitive to the initial configuration.

One of the goals of this investigation is to find well-behaved bounded solutions about $L_{4}$ to place a formation when solar gravitational force and vertical $(z, \dot{z})$ direction are considered, i.e., non-planar formations are carried out. From the results in Jorba and Villanueva (1997a,b), it is concluded that for each of the three periodic orbits shown in Fig. 1, there exists a Cantor family of two-dimensional tori that extend those vertical oscillations. In addition, for moderate vertical amplitudes, tori in theses families, that are close to the basic periodic orbits, have the same stability as these periodic orbits. Hence, most of the tori on the vertical families corresponding to the periodic orbits $\mathrm{PO} 2$ and $\mathrm{PO} 3$ are elliptic. Numerical experiments show that there exist stable regions around these vertical families, which correspond to non-escape trajectories from a neighborhood of one of those tori (Jorba, 2000).

In order to check the behavior of a formation in a bounded solution near triangular libration points in the restricted Sun-Earth-Moon scenario, Fig. 2 shows two quasi-periodic orbits (let us call QP2 and QP3) that fill densely one of the two dimensional tori on the families corresponding to the periodic orbits $\mathrm{PO} 2$ and PO3. The first plot in each row shows the $(x, y)$ projection, the second one contains the $(x, z)$ projection and the third one is the $(z, \dot{z})$ projection. The initial conditions of QP2 and QP3 are the same of the periodic solutions $\mathrm{PO} 2$ and $\mathrm{PO} 3$ with $z_{0}=0$ and $\dot{z}_{0}=0.05$. Due to the stability of these two trajectories about $L_{4}$, they will be chosen and analyzed as nominal trajectories for small formation flying. Numerical simulations

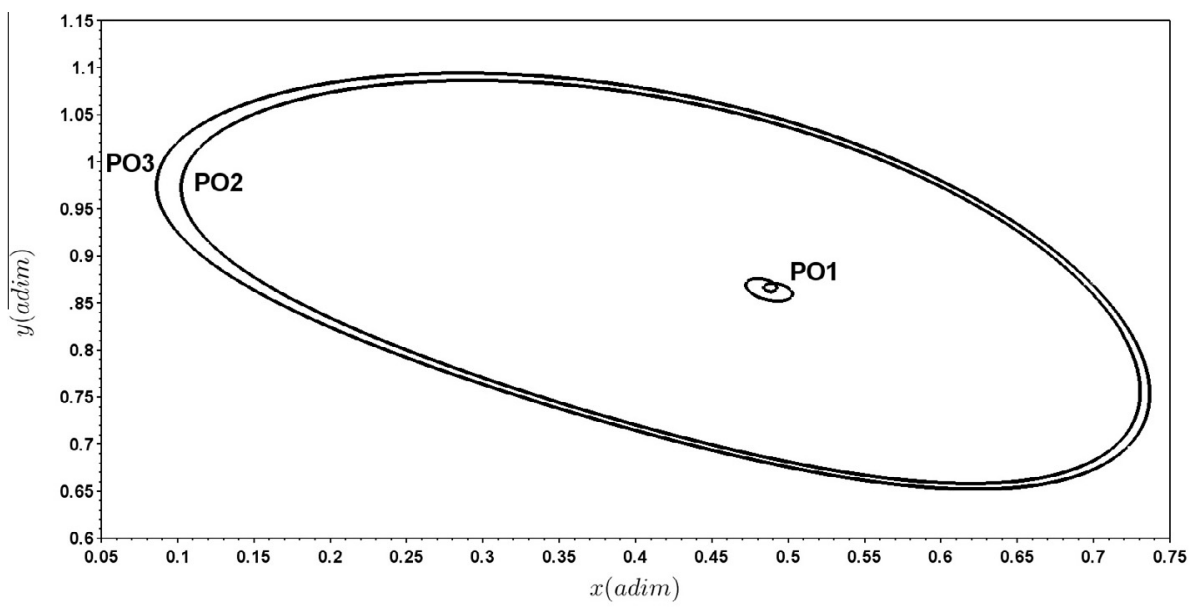

Fig. 1. Stable and unstable periodic orbits about $L_{4}$ in the Sun-Earth-Moon BCFBP with initial phase angle $\theta_{S}=0$ and period equal to the period of revolution of the Sun in the Earth-Moon synodical system. 


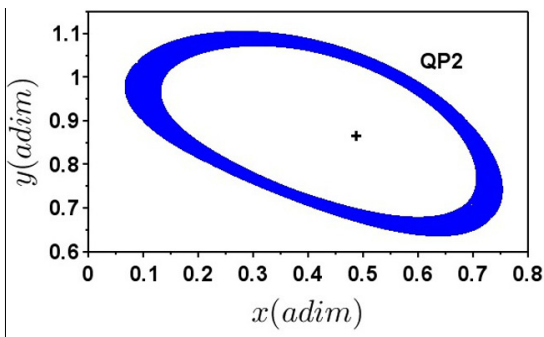

(a) $x-y$

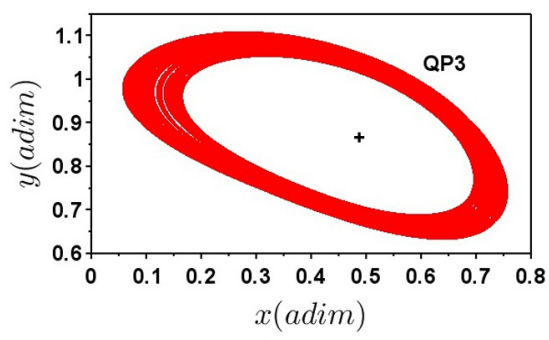

(d) $x-y$

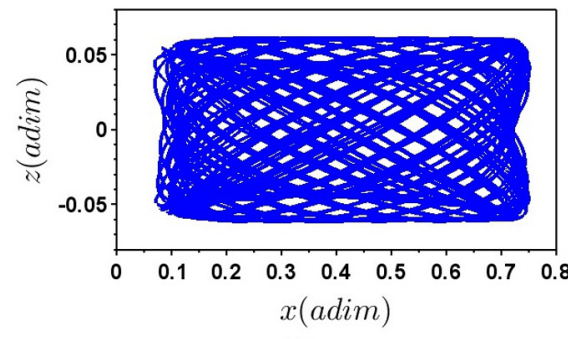

(b) $x-z$

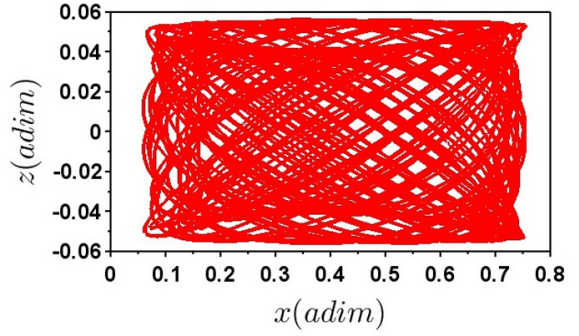

$(e) x-z$

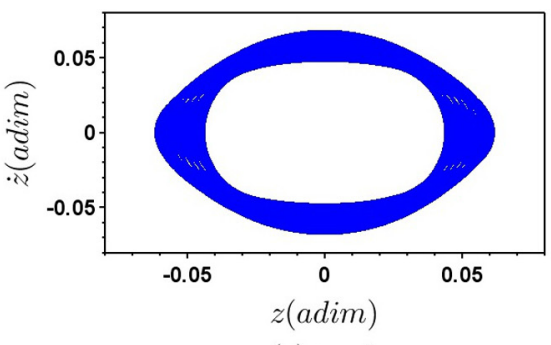

$(c) z-\dot{z}$

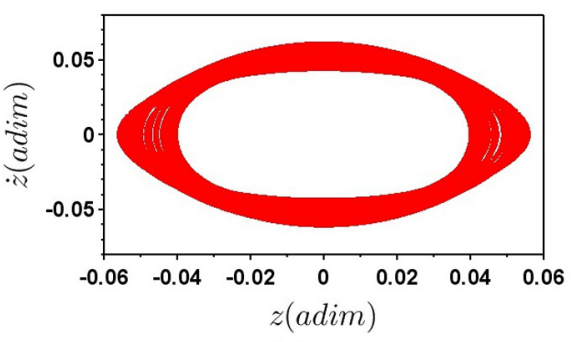

$(f) z-\dot{z}$

Fig. 2. Stable quasi-periodic orbits QP2 and QP3 that fill the $2-D$ tori corresponding to the periodic orbits PO2 and PO3, respectively, with initial phase angle $\theta_{S}=0$, and vertical direction $z_{0}=0$ and $\dot{z}_{0}=0.05$. First plot: $(x, y)$ projection; second plot $(x, z)$ projection; third plot $(z, \dot{z})$ projection. Integration time: 5 years.

have shown that a relevant part of the stable regions of the BCFBP persists for a very realistic model of the solar system, at least for 10 years and 1000 years in the case of the vertical families corresponding to $\mathrm{PO} 2$ and $\mathrm{PO} 3$, respectively (Jorba, 2000). Note that a stability time of 10 years could be enough for astronautical applications and there would be no need for any kind of control to keep a space mission near periodic orbit PO2. Furthermore, by real system we refer to the well-known Jet Propulsion Laboratory (JPL) ephemeris, file DE406, which specifies the past and future positions of the Sun, Moon, and nine planets in three-dimensional space. These data contain coefficients for Chebyshev polynomials (Press et al., 1992) that specify position (coordinates) and, by numerical differentiation, velocity components for each planet, including the Sun, Earth and Moon.

\section{Natural regions suitable for small formation}

For the purpose of this research, the satellite located at the center of the relative frame, will be called 'chief' satellite while the satellite operating in the vicinity of the chief will be called 'deputy' (see Fig. 3(a)). In order to avoid expansion or contraction with respect to the chief satellite, the existence of regions with zero relative velocity and zero relative radial acceleration (ZRRA) may be exploited (Gómez et al., 2001b; Perea et al., 2009). Assuming that the radius of the formation geometry structure (largest separation between spacecraft) is small, no greater than a few kilometers at most, then a linear approach gives all the relevant information about the local dynamics of the problem.

Let

$X=(x, y, z, \dot{x}, \dot{y}, \dot{z})^{T}$ be the vector that describes the position and velocity of the deputy satellite in the rotating frame, where superscript " $T$ " means transpose. Given a reference solution $X_{h}$ around $L_{4}$, i.e, the trajectory that is assumed to be followed by the chief satellite, then the linear variational equations of motion about the reference solution in matrix form is given by

$\delta \dot{X}(t)=A(t) \delta X(t)$,

where $\delta X(t)=X(t)-X_{h}(t)$ represents the deviation of the deputy satellite with respect to the chief's path. The coordinates of $\delta X(t)$ are defined in a coordinate system $(\hat{x}, \hat{y}, \hat{z})$ parallel to the rotating coordinate system $x, y, z$ and centered at $X_{h}(t)$ as shown in Fig. 3. The matrix $A(t)$ is time-varying of the form

$A(t)=\left(\begin{array}{cc}0_{3 \times 3} & I_{3 \times 3} \\ F & J\end{array}\right)$,

where the matrices $F$ and $J$ are defined as

$F=\left(\begin{array}{lll}\Omega_{x x} & \Omega_{x y} & \Omega_{x z} \\ \Omega_{y x} & \Omega_{y y} & \Omega_{y z} \\ \Omega_{z x} & \Omega_{z y} & \Omega_{z z}\end{array}\right), \quad J=\left(\begin{array}{ccc}0 & 2 & 0 \\ -2 & 0 & 0 \\ 0 & 0 & 0\end{array}\right)$.

The symbol $\Omega_{i j}$ stands for the partial derivatives $\partial^{2} \Omega / \partial i \partial j$ for $i, j=x, y, z$, and these partials are evaluated along the reference trajectory.

\section{Zero relative radial accelerations cones}

Writing the variations of the deputy satellite as $\delta X=(\delta r, \delta \dot{r})^{T}$, where $\delta r=(\delta x, \delta y, \delta z)^{T}$, the linear system (5) becomes 


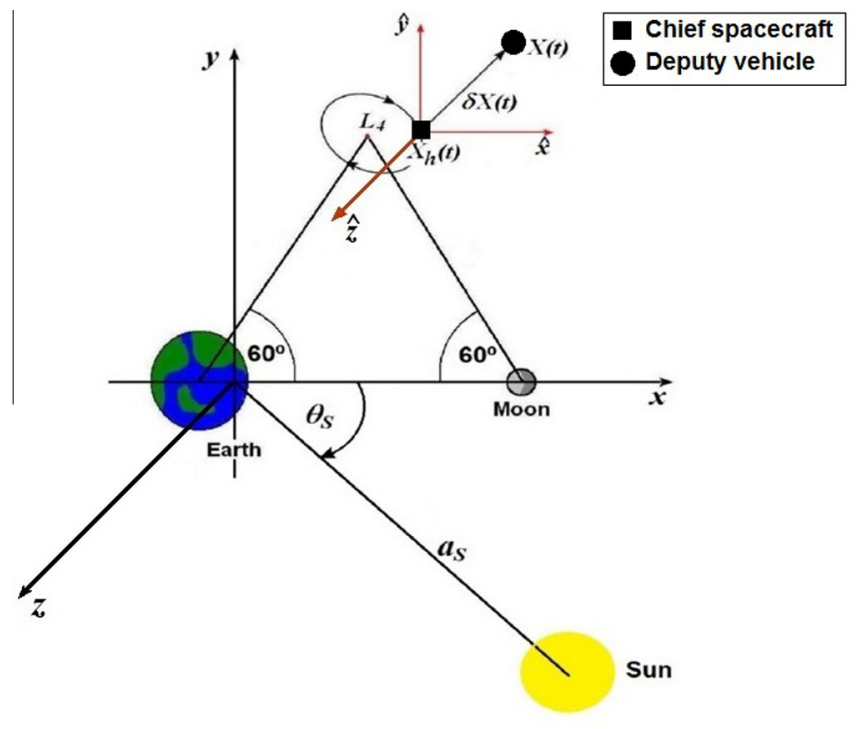

(a)

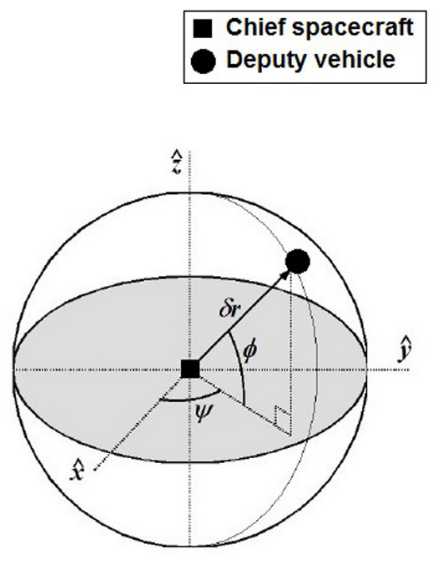

(b)

Fig. 3. (b) Illustration of a satellite formation flying about $L_{4}$ and its reference coordinate frame. (b) Sphere centered at the location of the chief satellite in the configuration space, such that, the velocity of all the points on the sphere are assumed to be equal to zero, and the locations of the deputy vehicle on the sphere are parametrized using spherical coordinates denoted by the angles $\psi$ and $\phi$.

$$
\left(\begin{array}{c}
\delta \dot{r} \\
\delta \ddot{r}
\end{array}\right)=\left(\begin{array}{cc}
0_{3 \times 3} & I_{3 \times 3} \\
F & J
\end{array}\right)\left(\begin{array}{l}
\delta r \\
\delta \dot{r}
\end{array}\right) .
$$

Since the distance between the deputy and chief satellites is small, we could assume that the relative velocity $\delta \dot{r}$ is equal to zero. From Eq. (8), the relative acceleration is then

$\delta \ddot{r}=F \delta r$.

Therefore, the locations with Zero relative radial acceleration component satisfy the equation

$\delta r^{T} F \delta r=0$.

Since the right hand side of Eq. (10) is equal to zero, then the ZRRA regions are represented by quadratics, that in general are elliptic cones. However, Salazar et al. (2015) showed that, for solutions far enough from Earth-Moon $L_{4}$ point, there exist certain locations in the selected path, where the ZRRA cones become intersecting planes. This fact produces a variation in the orientation of the cones surfaces along the reference trajectory as is shown below.

Regions with ZRRA component can also be computed numerically. Given a certain reference solution, consider a point along the trajectory that represents the state of the chief spacecraft. Now, centered at the location of the chief satellite, define a sphere of radius equal to $2 \mathrm{~km}$ in the configuration space, such that, the velocity of all the points on the sphere are assumed to be equal to zero (zero relative velocity condition). Using polar coordinates, the locations of the deputy vehicle on the sphere can be parametrized by the angles $\psi$ and $\phi$ as illustrated in Fig. 3(b). The relative acceleration $\delta \ddot{r}(\psi, \phi)$, that corresponds to each of the states of the sphere, can be evaluated using the right hand side of Eq. (1), whose dot product with $\delta r(\psi, \phi)$ will give the desired radial component.

In this way, since the analytical expression for the ZRRA derived by Eq. (10) represents a quadratic surface, Eq. (10) can be transformed into its canonical form using a change of coordinates. Because of matrix $F$ is symmetric, i.e., $F=F^{T}$, it can be diagonalized:

$F=P \Lambda P^{T}$,

where $P$ is the orthogonal matrix and $\Lambda$ is the real diagonal matrix, and are of the form

$P=\left(V_{1} V_{2} V_{3}\right), \quad \Lambda=\left(\begin{array}{ccc}\lambda_{1} & 0 & 0 \\ 0 & \lambda_{2} & 0 \\ 0 & 0 & \lambda_{3}\end{array}\right)$.

The real values $\lambda_{1}, \lambda_{2}$, and $\lambda_{3}$ represent the eigenvalues of $F$. The column vectors $V_{1}, V_{2}$, and $V_{3}$ denote the eigenvectors of $F$ and form an orthonormal basis that identifies the principal directions of the quadratic surface.

Now, defining the vector $\delta \bar{r}=(\bar{x}, \bar{y}, \bar{z})^{T}$, such that, $\delta \bar{r}=P^{T} \delta r$, then Eq. (10) can be rewritten in the following form

$\lambda_{1} \bar{x}^{2}+\lambda_{2} \bar{y}^{2}+\lambda_{3} \bar{z}^{2}=0$.

Eq. (13) represents a second order surface, whose orientation also represents the principal directions corresponding to the cones and, therefore, depends on the sign of the eigenvalues $\lambda_{1}, \lambda_{2}$, and $\lambda_{3}$ (Hilbert and Cohn-Vossen, 1999). To investigate the effect of the reference path on the quadratic surfaces, Fig. 4 shows the dynamical evolution of the eigenvalues of $F$ along the quasi-periodic solutions QP2 $(a-c)$ and QP3 $(d-f)$, during one period of 
revolution of the Sun (about 29 days). As can be seen in Fig. 4, eigenvalues $\lambda_{1}$ and $\lambda_{3}$ are always negative and positive, respectively. However, there are two locations where $\lambda_{2}$ approaches zero. Hence, at these positions the cones become intersecting planes and, at once, a change in the orientation of the cones occurs, as illustrated in Fig. 5. This dynamical evolution is repeated along the quasi-periodic solutions QP2 and QP3.

To help in the interpretation of the orientation of the quadratic surfaces, Fig. 6 shows the $(x, y)$ projection in the rotating frame of the three principal directions $\left\{V_{1}, V_{2}, V_{3}\right\}$ along the two reference solutions during 29 days. Notice that a close proximity of the Earth leads to a higher level of sensitivity and, eventually, to a change in the orientation of the zero natural drift regions. In particular, the two locations at time $t=10$ days and $t=25$ days in Fig. 6(a) and (b), respectively, with $\lambda_{2}<0$, represent the closest distance with the Earth. Over the time interval that surrounds those times in Fig. 4, that is the time corresponding to the close proximity of the Earth, the magnitude (absolute value) of the three eigenvalues of $F$ gets larger. This fact implies that, when the chief spacecraft is passing close to the Earth, the relative velocity and relative acceleration between the spacecraft are higher and, therefore, the cost to maintain the deputy vehicle within the Zero relative radial acceleration cones is higher as shown in the next

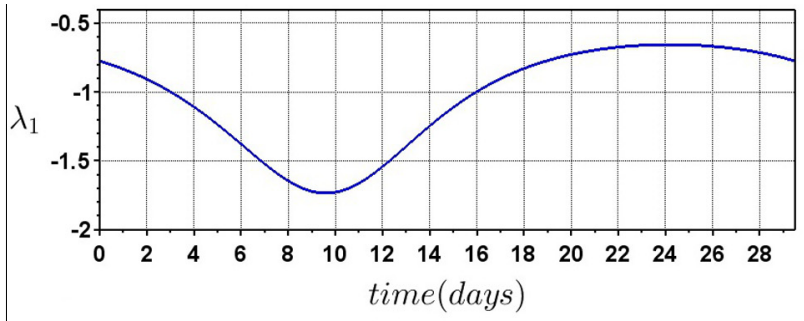

(a)

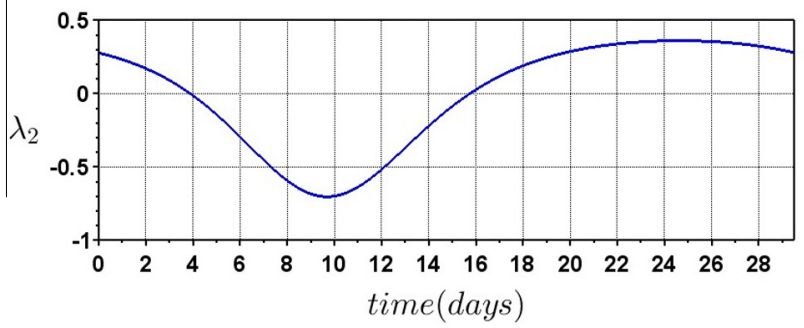

(b)

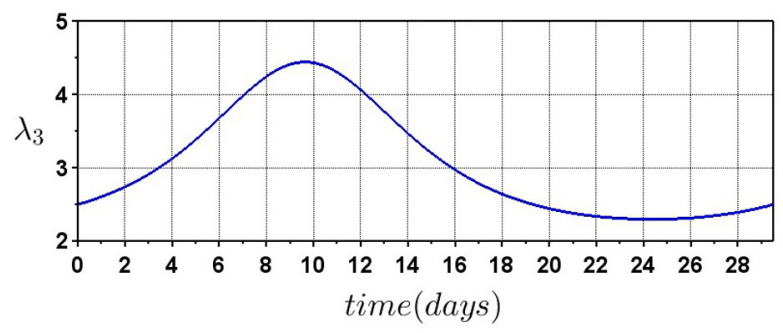

(c) section. Finally, the two locations at time $t=24$ days and $t=10$ days in Fig. 6(a) and (b), respectively, with $\lambda_{2}>0$, represent the farthest distance to the Earth.

To illustrate how the relative radial acceleration varies with respect to the relative directions on a sphere, the relative acceleration between the chief and deputy is computed numerically at previous locations ( $t=4$ days, 10 days, 16 days, 24 days) along the reference trajectory QP2. The sphere of points corresponds to a radius of $2 \mathrm{~km}$, and the test points are parametrized by the two angles, an in-plane angle $\psi$ measured from the $\hat{x}$-axis and an out-of-plane angle $\beta$, as illustrated in Fig. 3(b). Due to the linear approach, the velocity of all the points on the sphere is assumed to be equal to the velocity of the selected location on the reference trajectory. The surface of relative radial acceleration at time $t=4$ days (a), 10 days (b), 16 days (c) and 24 days (d) along the reference trajectory QP2 is presented in Fig. 7. These surfaces posses two minima located at about $\psi=150^{\circ}$ and $\phi=0^{\circ}$, and two maxima located at about $\psi=60^{\circ}$ and $\phi=0^{\circ}$. The contour lines on the surfaces reflect the value of the relative radial acceleration at the specific locations on the sphere. The dash-dotted green contour lines near the two minima, correspond to the zero drift regions along the cones surfaces found analytically using the linear approach. Similarly, the magnitude of the maximum radial

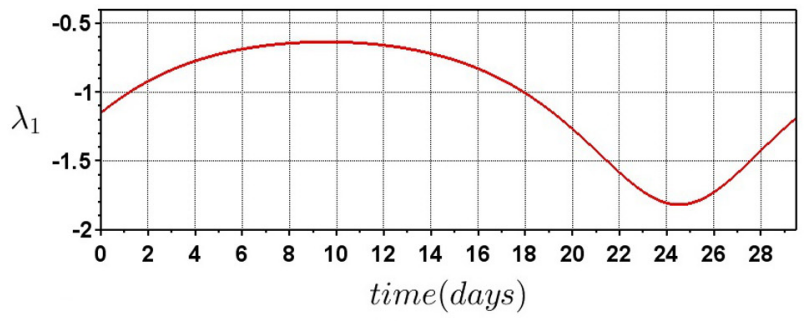

(d)

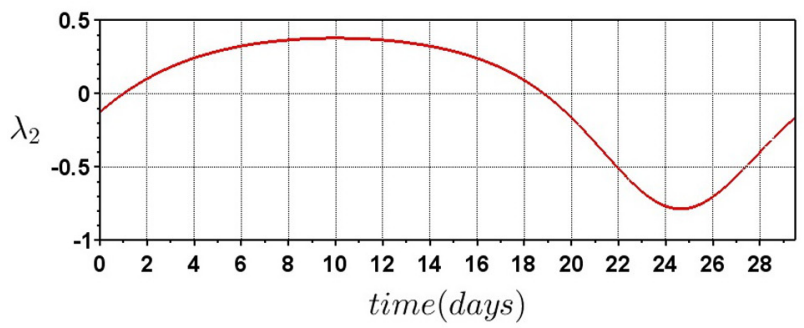

(e)

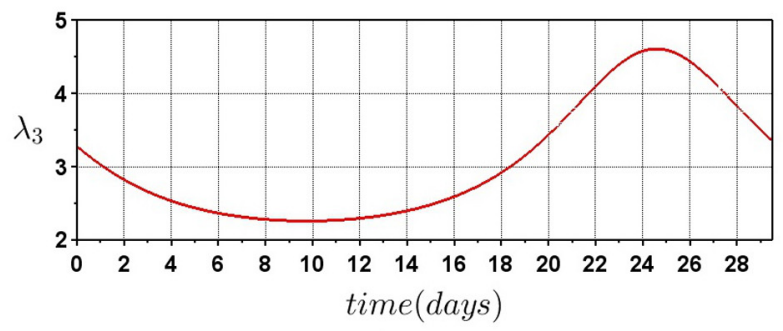

$(f)$

Fig. 4. Eigenvalues of $F$ along the quasi-periodic solutions QP2 $(a-c)$ and QP3 $(d-f)$, during one period of revolution of the Sun (about 29 days). 

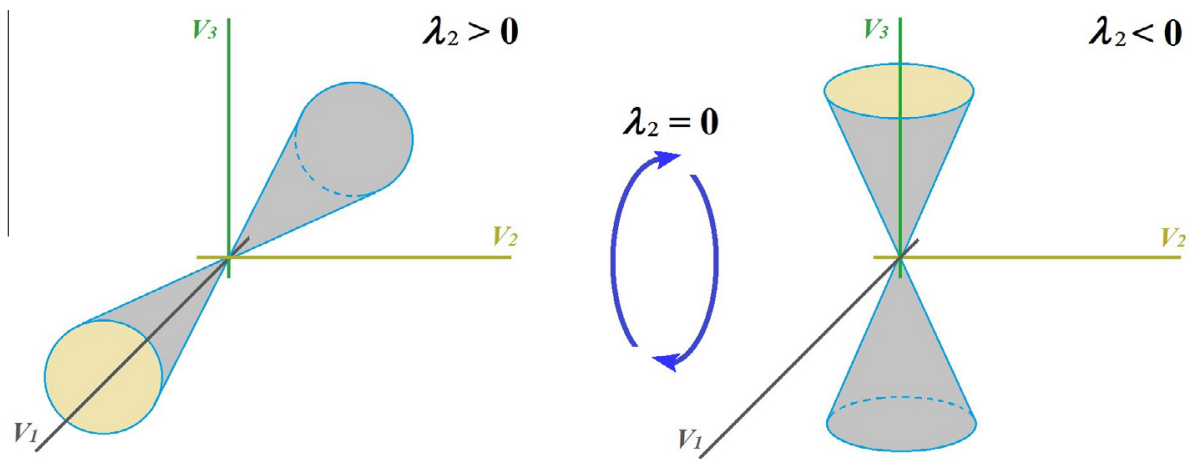

Fig. 5. Illustration of the change in the cones' orientation when $\lambda_{2}=0$.

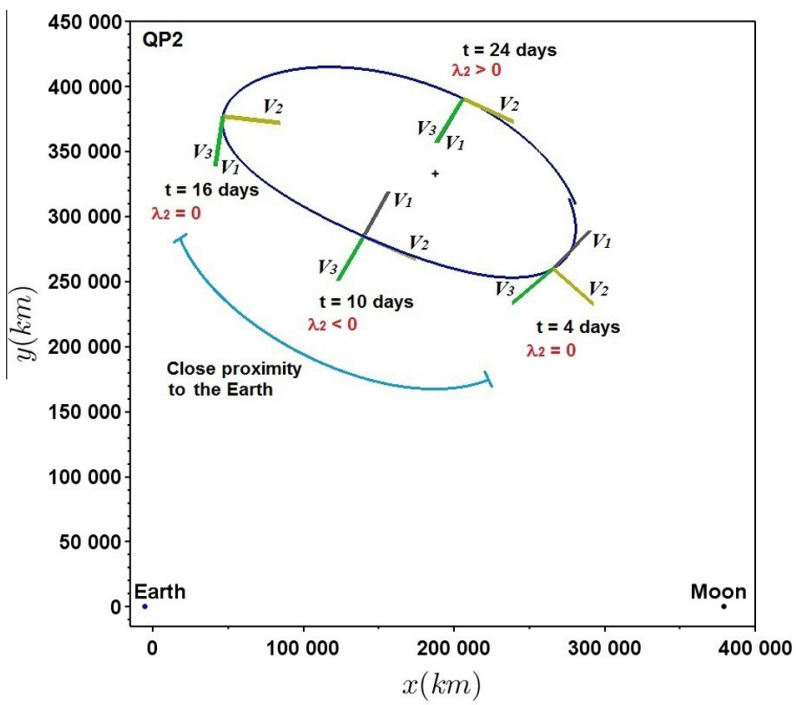

(a)

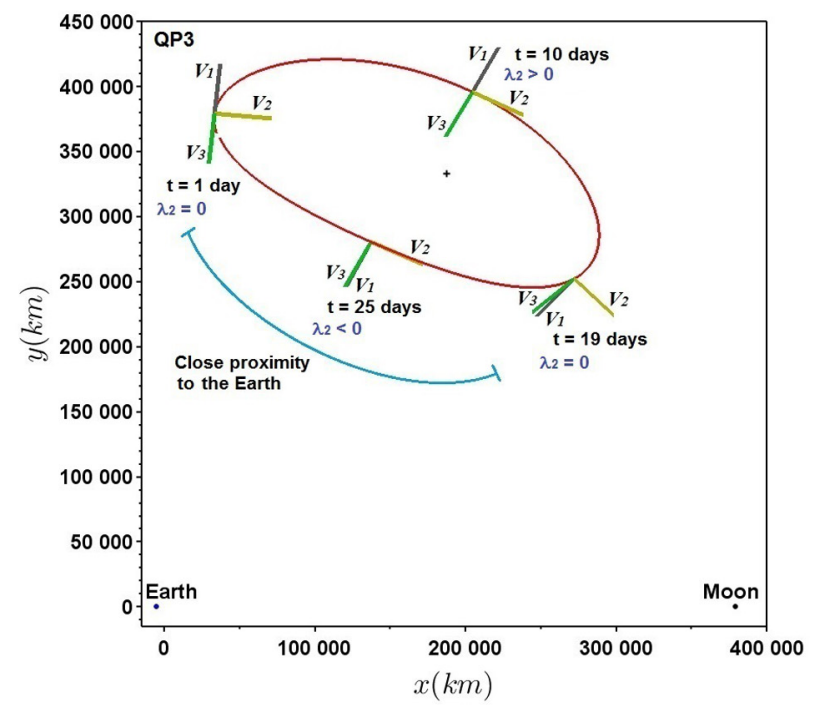

(b)

Fig. 6. Principal directions $\left\{V_{1}, V_{2}, V_{3}\right\}$ along the two reference solutions QP2 $(a)$ and QP3 $(b)$ during 29 days.

acceleration is larger at $t=10$ days, and lower at $t=24$ days, corresponding to the closest and farthest distance to the Earth, respectively.

Note that the relative position vectors with $\psi=60^{\circ}$ and $\phi=0^{\circ}$, and $\psi=150^{\circ}$ and $\phi=0^{\circ}$ point in the same direction as the lines that connect the libration point $L_{4}$ with the Earth and the Moon, respectively. These two vectors are orthogonal. Therefore, as shown in Figs. 6 and 7, not only the close proximity of the Earth produces a higher drift, but also the relative position vector of the deputy satellite with respect to the chief spacecraft points in the same direction that the vector that connects $L_{4}$ with the Earth's center. To compare the natural drift between the two spacecraft along the reference trajectory QP2, a deputy vehicle is placed on the sphere centered at the chief satellite with radius of $2 \mathrm{~km}$, and the previous locations ( $t=4$ days, 10 days, 16 days, 24 days) represent the initial position of the chief satellite. Subsequently, a set of points on the sphere is obtained, and the deputy vehicle is initially placed on the surface of the sphere. The initial velocity of the spacecraft is assumed to be the same. Then, both paths are integrated and the natural drift is computed after 5 days. The surface of natural drift as a function of the two initial angles $\psi$ and $\phi$ appears in Fig. 8. Similarly, the surfaces of natural drift posses two maxima and two minima that correspond to the same locations as the maxima and minima for the relative radial acceleration illustrated in Fig. 7. The magnitude of the maximum natural drift is also larger at $t=10$ days (about $8 \mathrm{~km}$ ), and lower at $t=24$ days (about $3 \mathrm{~km}$ ). Therefore, the amount of natural drift depends on the initial position selected for the chief satellite, and it is higher when the chief satellite is passing close to the Earth. However, Fig. 8 shows that it is possible to reduce the variation of the mutual distance if the deputy vehicle is placed on the zero radial acceleration cones, which in principle, will keep fixed the mutual distance within the formation. Additionally, a linear behavior between the natural drift and the initial radius of the formation was found, i.e., if the initial radius is doubled, then the natural drift after 5 days is also multiplied by 2 . The cost to maintain a formation within the zero drift surfaces is considered in the next section. 


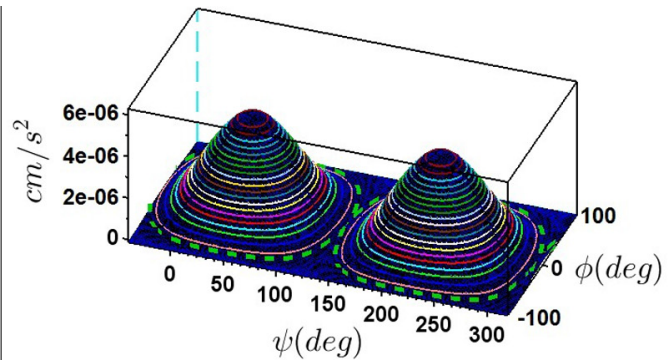

(a)

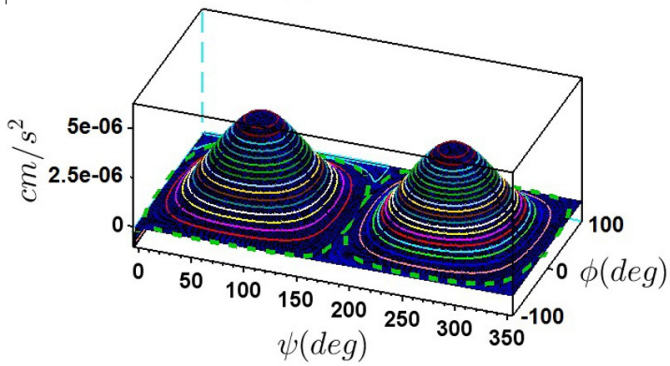

(c)

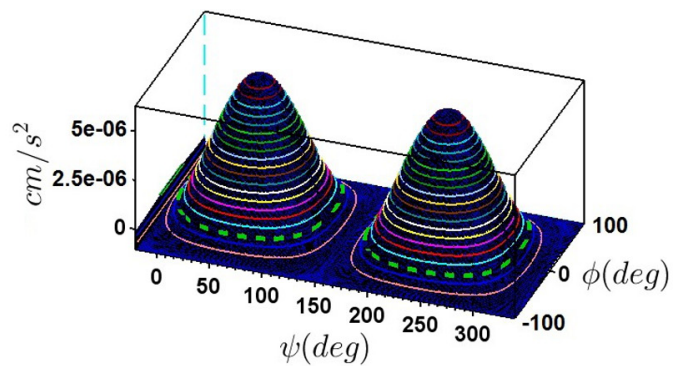

(b)

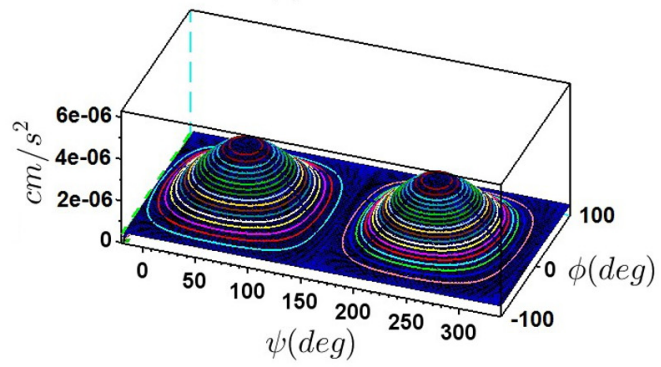

(d)

Fig. 7. Surface of the relative radial acceleration between the spacecraft at time $t=4$ days (a), 10 days (b), 16 days (c), 24 days (c), along the reference trajectory QP2. (For interpretation of the references to color in this figure legend, the reader is referred to the web version of this paper.)

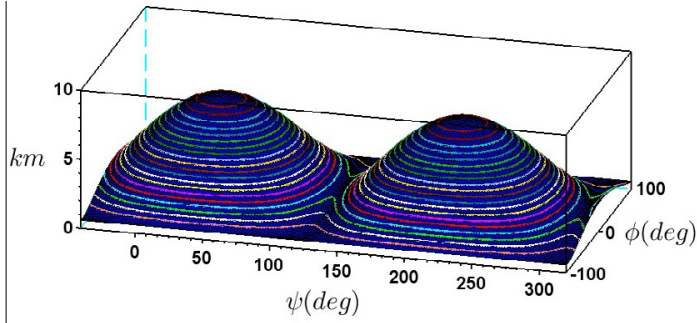

(a)

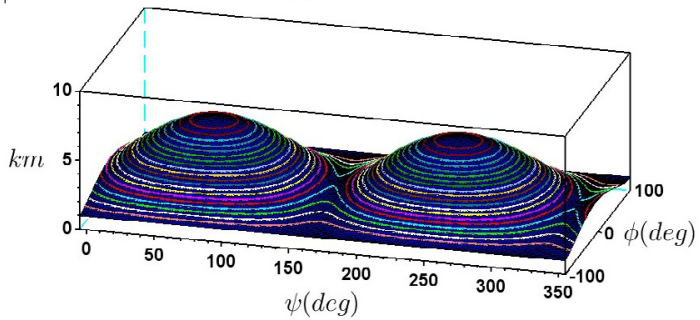

(c)

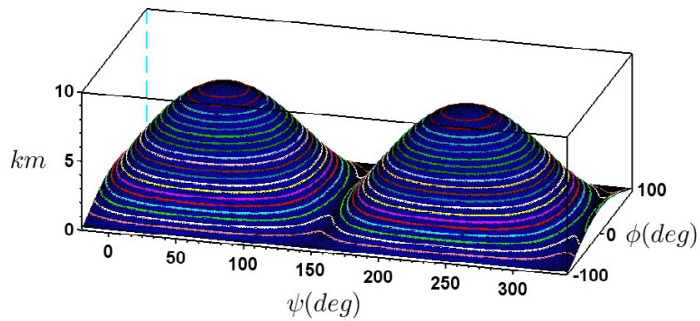

(b)

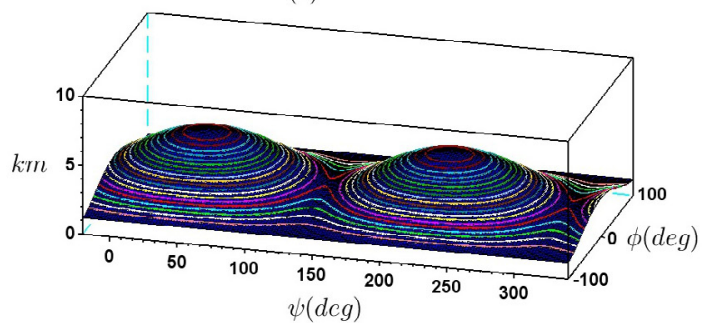

(d)

Fig. 8. Surface of natural drift after 5 days for a 2-km formation at initial positions $t=4$ days (a), 10 days (b), 16 days (c), 24 days (d) along the two reference solutions QP2.

\section{Controlling motions within the ZRRA cones}

In this last section, we will show some results related to the control of a formation moving on the zero radial acceleration cones. Two different control strategies have been employed: a bang-off-bang control and a minimum weighted total $\Delta V$ consumption.

For a given formation size and a given time of integration, the relative position of the deputy vehicle on a generatrix of a cone associated with the reference trajectory is defined by the angle $\theta$, as illustrated in Fig. 9(a). This figure shows a generatrix that moves around the principal direction $V_{1}$. However, because there exists a change in the orientation of the cones when the eigenvalue $\lambda_{2}$ approaches zero (see Fig. 5), then the quadratic surface defined by Eq. (13) is not satisfied in the real domain for every angle $\theta$. Hence, the endpoints of the intervals for $\theta$, such that Eq. (13) is satisfied, can be approximated by the inclination of the intersecting planes when $\lambda_{2}\left(t^{*}\right)=0$. In this case, the slope is equal to $\pm \sqrt{\lambda_{1}\left(t^{*}\right) / \lambda_{3}\left(t^{*}\right)}$. Thus, it was found that Eq. (13) is satisfied along the reference trajectories QP2 and QP3 for $25^{\circ}<\theta<155^{\circ}$ and $205^{\circ}<\theta<335^{\circ}$. These intervals are located in the region colored in pink in Fig. 9(b). 


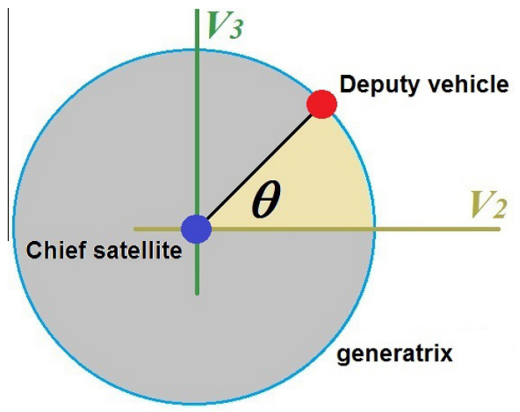

(a)

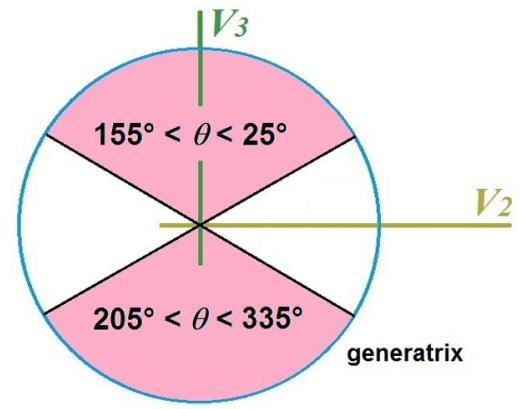

(b)

Fig. 9. (a) Generatrix of a cone defined by the angle $\theta$ and associated with the reference trajectory. (b) Intervals for angle $\theta$ (pink region) such that the quadratic surface defined by Eq. (13) is satisfied. (For interpretation of the references to color in this figure legend, the reader is referred to the web version of this paper.)

For both control strategies several parametric studies have been done, considering different geometrical possibilities, such as parallel and non-parallel translations between ZRRA cones. In the parallel translations, the initial and final generatrices $\theta$ are equal, and in the non-parallel ones, they are different.

\subsection{The bang-off-bang control}

Due to the symmetry of the cones, only one generatrix is taken on each cone, assuming a formation of two satellites: the deputy vehicle is situated at the end of the cone's generatrix associated with the reference orbit, while the chief spacecraft, situated at the cone's vertex, moves along the reference solution without any control acting on it. As the chief satellite moves along the orbit after some $\Delta t$, the deputy vehicle is forced to be always on a generatrix of a ZRRA cone, keeping their mutual distance. This situation is illustrated in Fig. 10 for four deputy vehicles placed initially on different generatrices of the cones. The chief satellite is placed in different initial locations, and two impulse maneuvers are applied in each deputy vehicle during the time displacement, in such a way that, the spacecraft are placed finally on the corresponding ZRRA cone in the same amount of time. Note that, even if the initial configuration is within the zero drift regions, drift between the two spacecraft still occurs, thus the relative position vector of the deputy vehicle is not, in general, on any generatrix of any ZRRA cone.

Let us denote by $\delta X_{0}, \delta X_{f}$ the initial and final states (position and velocity), respectively, of the deputy vehicle after some $\Delta t$. If $\Delta V_{0}, \Delta V_{1}$ represent the two impulse maneuvers to be applied at $t=t_{0}$ and $t=\alpha \Delta t, 0<\alpha \leqslant 1$, respectively, and $\Phi\left(t, t_{0}\right)$ the state-transition matrix of the linear system (5), then the equations that must be solved for the computation of the impulse translation maneuvers are

$\Phi(\Delta t, \alpha \Delta t)\left(\Phi\left(\alpha \Delta t, t_{0}\right)\left(\delta X_{0}+\left(\begin{array}{c}0 \\ \Delta V_{0}\end{array}\right)\right)+\left(\begin{array}{c}0 \\ \Delta V_{1}\end{array}\right)\right)=\delta X_{f}$

If we fix the parameter $\alpha$, the linear system (14) will have six equations and six unknowns: the components of the two impulses. Thus, the total cost of the translation maneuvers

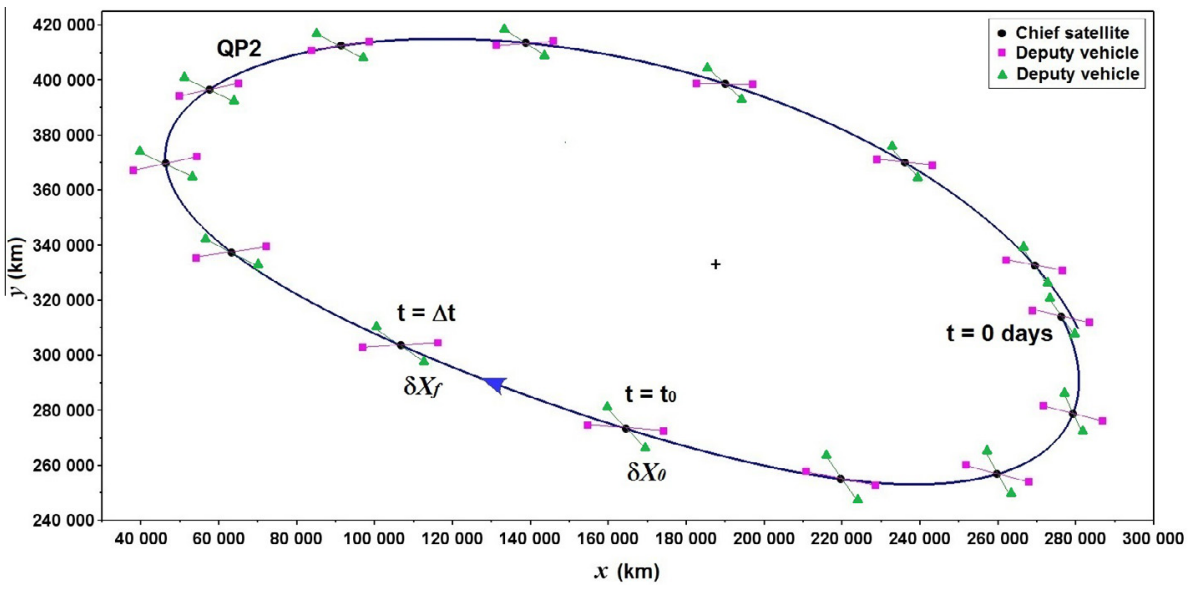

Fig. 10. A formation of four deputy vehicles moving along the reference solution QP2. The chief satellite is placed in different initial locations, and after a some $\Delta t$, the deputy vehicles are forced to be always on a generatrix of a ZRRA cone, keeping their mutual distance. 
will be $\left\|\Delta V_{0}\right\|+\left\|\Delta V_{1}\right\|$. Although the cost depends on the parameter $\alpha$ fixed, no significant variation of this magnitude with $\alpha$ was found. The results presented in this section were obtained with $\alpha=1$. Fig. 11 shows the total cost $(\mathrm{cm} / \mathrm{s})$ for the deputy vehicle, of parallel translation maneuvers, i.e., $\theta\left(t_{0}\right)=\theta(\Delta t)=120^{\circ}$, for $\Delta t=1.5,3$, and 4.5 days, when the vertex of the departure cone moves along the bounded solutions QP2 and QP3, and the distance between the spacecraft placed initially and finally on the ZRRA cones is equal to $2 \mathrm{~km}$. The horizontal axis on Fig. 11 represents the locations of the initial position of the chief satellite. The point 0 denotes the initial conditions of solutions QP2 and QP3, and the point 1 the position after one period of revolution of the Sun (about 29 days). Note that, as was stated in the previous section and shown in Figs. 7 and 8, the maximum total cost corresponds to the closer point to the Earth (see Fig. 6), for any value of $\Delta t$. Additionally, the cost of the transfer behaves almost linearly with the time displacement $\Delta t$.

Now, we are interested in exploring the cost of parallel translations as a function of the generatrix along the departure cone. Thus, fixing the vertex of departure at the locations that are identified in the closest distance to the Earth of the bounded solutions QP2 and QP3 (see Fig. 6), Fig. 12 shows that, for any value of $\Delta t$, the maximum cost is obtained when the departure generatrix $\theta\left(t_{0}\right)$ is equal to $90^{\circ}$ and $270^{\circ}$. On the other hand, the results given in Fig. 12 suggest that the best configurations for the formation occurs for the extremes of the intervals for $\theta$ (see Fig. 9(b)). These four departure generatrices, as well as those of maximum cost, will be used in the next section.

For the second exploration, we have also studied the non-parallel translation maneuvers, i.e., the initial and final configurations of the formation are on different generatrices of the ZRRA cone, i.e., $\theta\left(t_{0}\right) \neq \theta(\Delta t)$. In the first case, the departure generatrix is fixed with $\theta\left(t_{0}\right)=25^{\circ}$, and the arrival one is varied within the intervals for $\theta$, at a distance $\Delta t$ from the first. The results corresponding to these transfer maneuvers appear in Fig. 13. When the initial configuration is almost parallel to the final one $\left(\theta\left(t_{0}\right) \approx \theta(\Delta t)\right)$ the cost of the transfer is minimum, independently of the value of $\Delta t$ or the position of the initial configuration along the quasi-periodic solutions, as shown in Fig. 13(b). Additionally, the cost increases as $\Delta t$ decreases. However, this situation reverses for angles close to $25^{\circ}$.

Finally, in the second case, the transfers from an arbitrary departure generatrix to an arbitrary arrival generatrix of two ZRRA cones are studied. Fig. 14 shows the corresponding total transfer cost $(\mathrm{cm} / \mathrm{s})$ for these kind of maneuvers. From this figure, we can see that the cost surfaces reach their minima on the in-plane diagonal. Therefore, the transfer costs are minimum when both the initial and final generatrix are almost parallel.

\subsection{The minimum $\Delta V$ control strategy}

Similarly, given an initial state $\delta X_{0}$ at $t=t_{0}$, the goal of this control procedure is to reach a final state $\delta X_{f}$ at $t=t_{N}$ $(N \geqslant 2)$, applying a sequence of correction maneuvers $\Delta V_{0}, \Delta V_{1}, \ldots, \Delta V_{N-1}$ at certain epochs $t_{0}, t_{1}, \ldots, t_{N-1}$, and executed at uniformly distributed instants, i.e, $t_{i+1}-t_{i}=$ constant. Therefore, assuming that the maneuvers are performed without errors, they should satisfy the following linear constraint:

$$
\Phi\left(t_{N}, t_{N-1}\right)\left(\cdots \Phi\left(t_{1}, t_{0}\right)\left(\delta X_{0}+\left(\begin{array}{c}
0 \\
\Delta V_{0}
\end{array}\right)\right)+\cdots+\left(\begin{array}{c}
0 \\
\Delta V_{N-1}
\end{array}\right)\right)=\delta X_{f} .
$$

Since there are infinitely many different values of $\Delta V_{0}, \Delta V_{1}, \ldots, \Delta V_{N-1}$ that satisfy the linear system (15), we select those that minimize the cost function

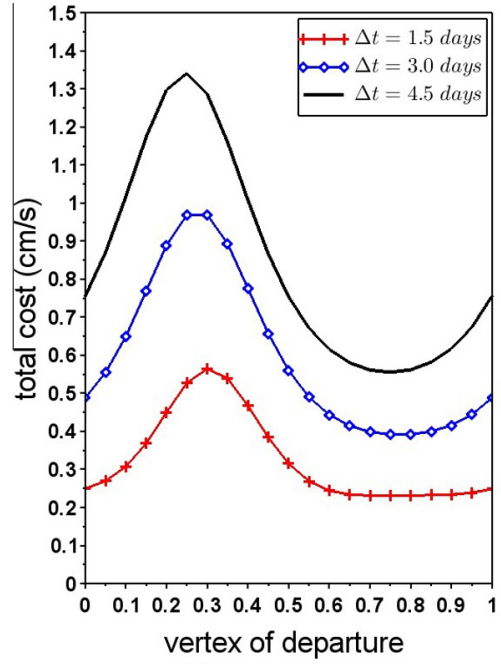

(a)

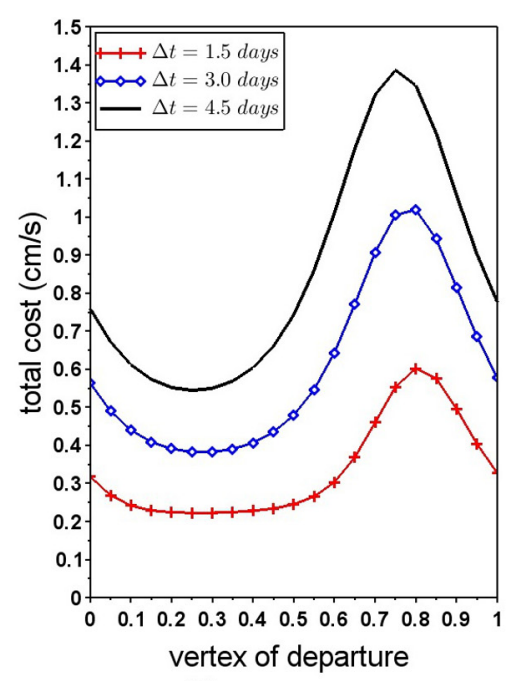

(b)

Fig. 11. Total cost $(\mathrm{cm} / \mathrm{s})$ of parallel translation maneuvers with $\theta\left(t_{0}\right)=120^{\circ}$ for $\Delta t=1.5,3$, and 4.5 days, when the vertex of the departure cone moves along the bounded solutions QP2 (a) and QP3 (b), and the distance between the spacecraft on the initial and final ZRRA cones is of $2 \mathrm{~km}$. 


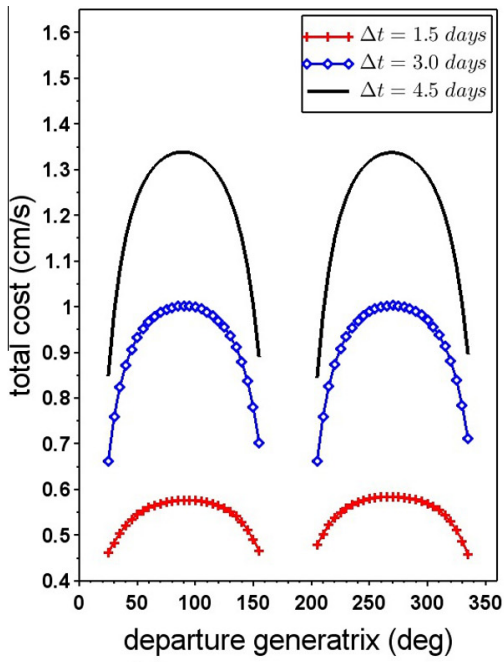

(a)

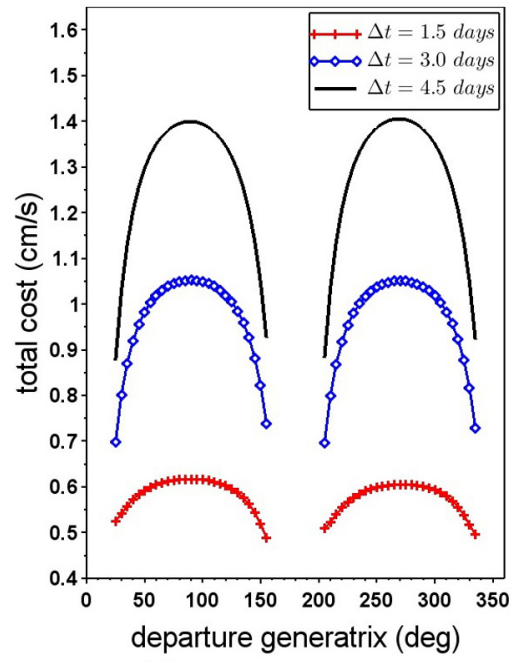

(b)

Fig. 12. Total transfer cost $(\mathrm{cm} / \mathrm{s})$ of parallel translation maneuvers for $\Delta t=1.5,3$, and 4.5 days, fixing the vertex of departure at the locations that are identified in the closest distance to the Earth of the bounded solutions QP2 (a) and QP3 (b) (see Fig. 6).

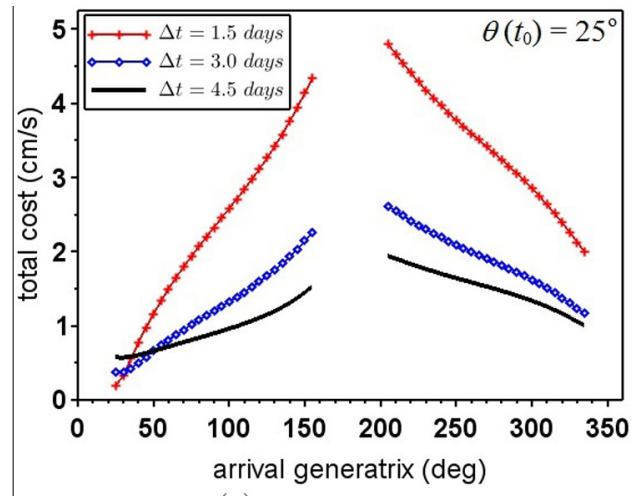

(a)

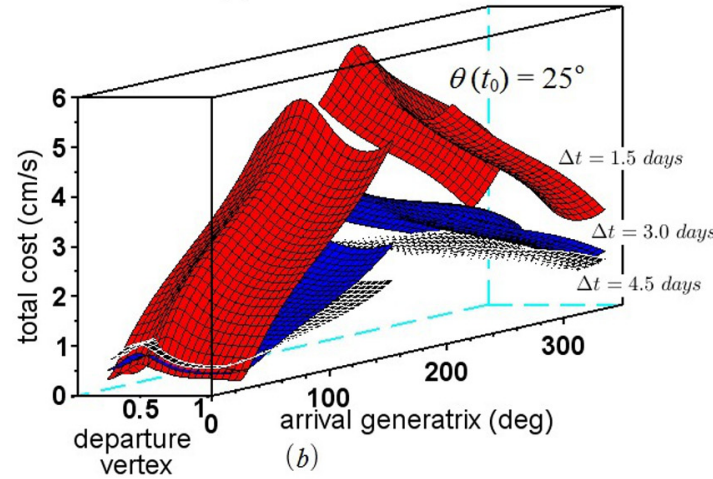

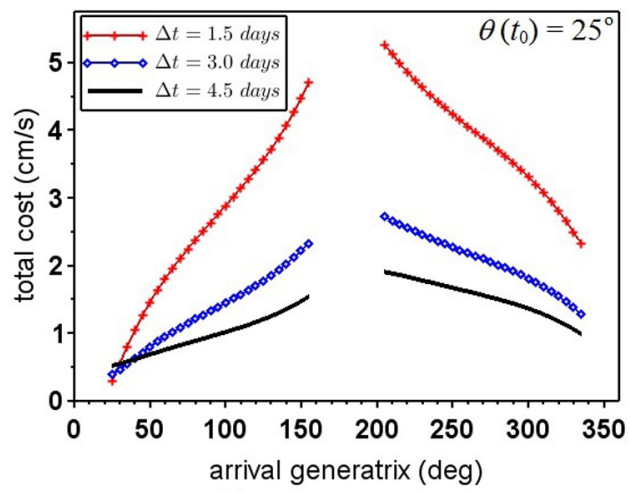

(c)

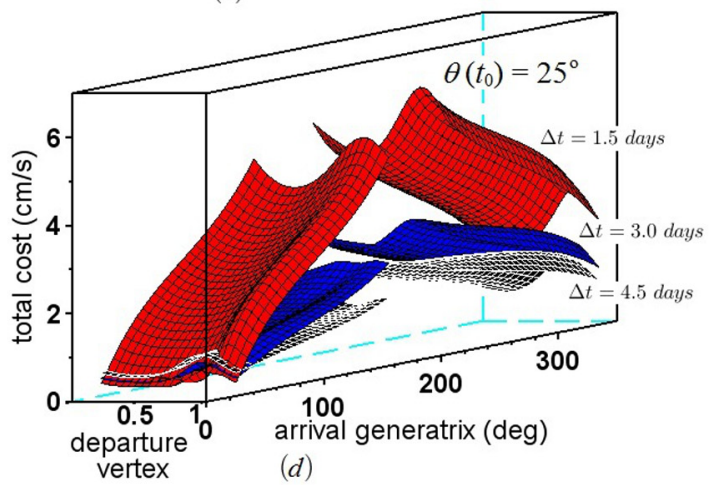

Fig. 13. Total transfer cost $(\mathrm{cm} / \mathrm{s})$ of non-parallel translation maneuvers for $\Delta t=1.5,3$, and 4.5 days, fixing the departure generatrix with $\theta\left(t_{0}\right)=25^{\circ}$ in the bounded solutions QP2 (a), (b) and QP3 (c), (d).

$\sum_{i=0}^{N-1} q_{i}\left\|\Delta V_{i}\right\|^{2}$,

where $q_{0}, q_{1}, \ldots, q_{N-1}$ are weights which must be fixed in advance. In this work, we have used $q_{i}=2^{-i}$, so the magnitude of the first impulsive maneuvers remain small and, therefore, the last impulsive maneuvers will practically position the deputy vehicle on the ZRRA cone at $t=t_{N}$. Because the cost function (16) is quadratic and the constrain (15) is linear, the minimum weight total $\Delta V$ consumption problem can be solved easily by using Lagrange multipliers.

Since parallel translation maneuvers are cheaper than non-parallel, as was shown previously, we only consider parallel translations in this section. We have analyzed the total magnitude of the controls applied, which is the sum of the magnitudes of all the control maneuvers, as a function of the generatrix along the departure cone, the time interval $t_{N}$ required by the spacecraft recover the 

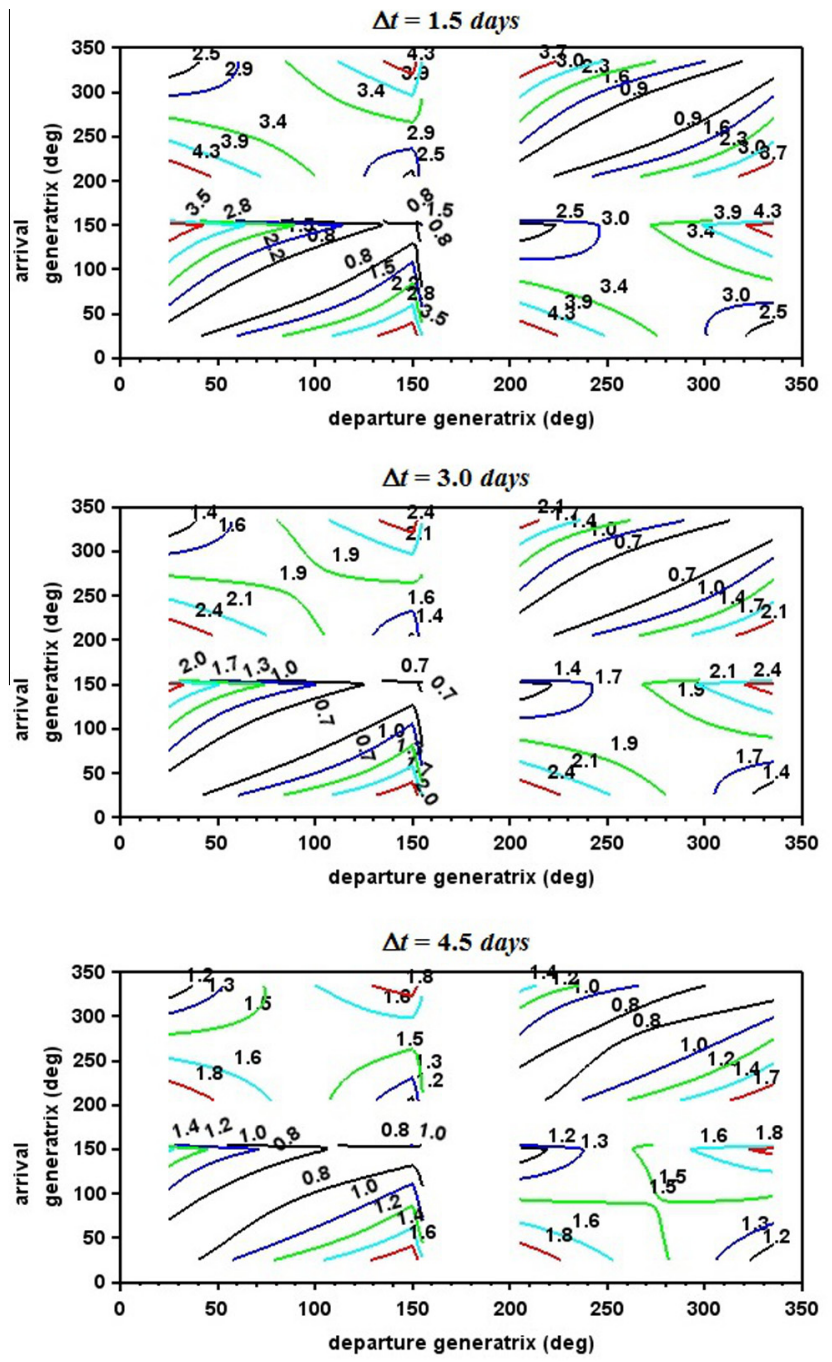
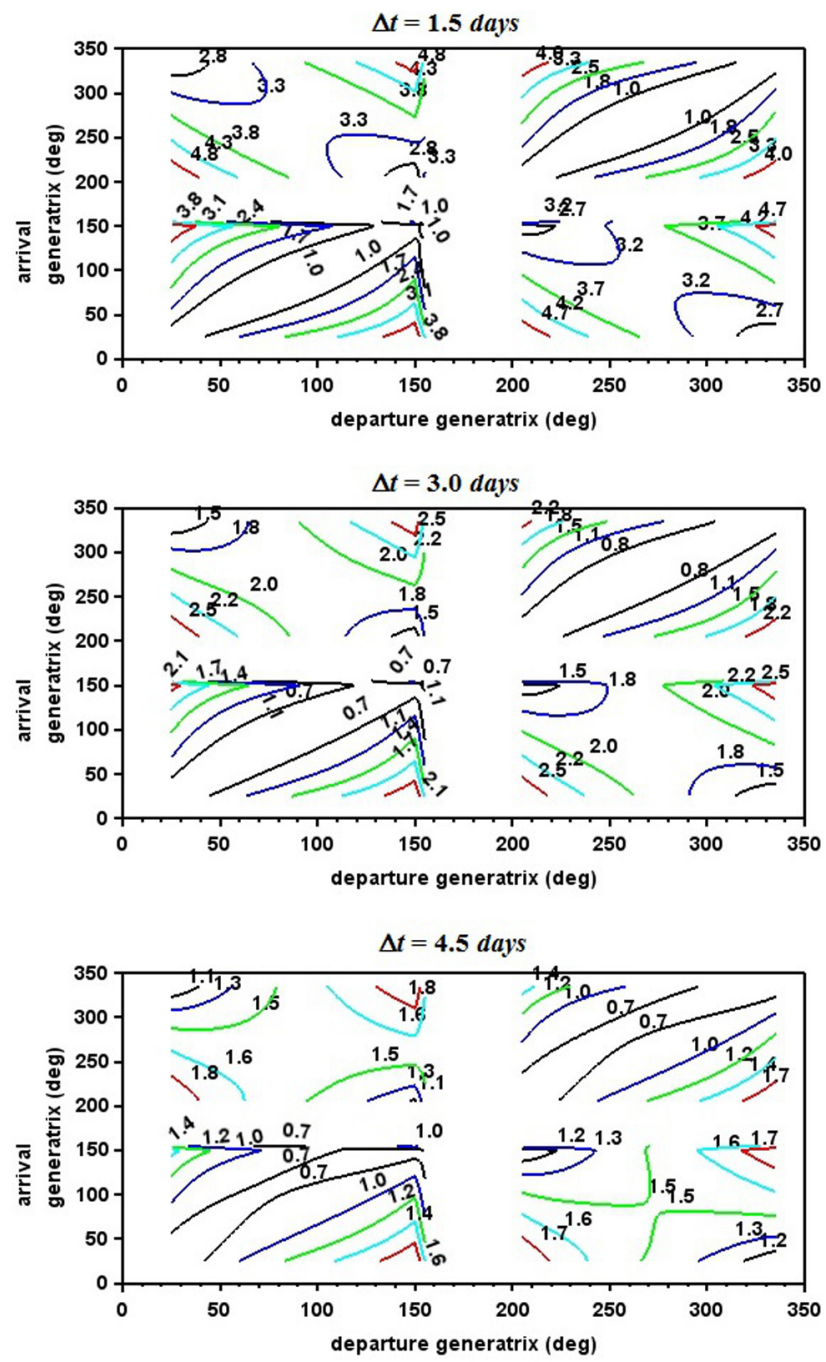

Fig. 14. Total transfer cost $(\mathrm{cm} / \mathrm{s})$ of non-parallel translation maneuvers for $\Delta t=1.5,3$, and 4.5 days, between arbitrary departure and arrival generatrices of two fixed cones in the bounded solutions QP2 (left column) and QP3 (right column).

formation, and the number $N$ of correction maneuvers. Thus, Figs. 15 and 16 present the magnitude of the correction maneuvers as a function of time for different sets of values of the time interval $t_{N}$ and the number of maneuvers $N \geqslant 2$, when the chief satellite moves along the reference solutions QP2 and QP3, respectively. For each simulation, the distance between the spacecraft placed initially and finally on the ZRRA cones is $2 \mathrm{~km}$, and the angle of the generatrix $\theta\left(t_{N}\right)=25^{\circ}$.

The results presented in Figs. 15 and 16 show that the magnitude of the first maneuvers remain small due to the weights chosen in this study. On the other hand, the magnitude of the control maneuvers reaches a maximum value in the locations where the formation is closer to the Earth (see Figs. 6 and 11). Now, notice that keeping fixed the time interval $t_{N}$, the magnitude of each $\Delta V_{i}$ maneuver decays approximately as $2^{-(N-2) / 2}$ with regards to the number of maneuvers $N \geqslant 2$. On the other hand, there exists a small reduction in the magnitude of the control maneuvers when the time interval increases if the number of maneuvers remains fixed.

Furthermore, Fig. 17 presents the total transfer cost during 1 year for different sets of values of the angle of the generatrix $\theta\left(t_{N}\right)$, the time interval $t_{N}$ and the number of maneuvers $N \geqslant 2$. The results obtained for a radius of $2 \mathrm{~km}$, found two local minima of the total cost when the angle $\theta\left(t_{N}\right)$ in the ZRRA cone is close to $90^{\circ}$ and $270^{\circ}$. Note that, with regards to the bang-off-bang control, these angles correspond to the points where the total cost reached a maximum value (see Fig. 12). In addition, varying the time interval and the number of the control maneuvers, and keeping fixed the departure generatrix, the best results are obtained when $t_{N}=4 \mathrm{~h}$ and $N=3$. Finally, it was found that if the distance between the spacecraft placed on the ZRRA cone is doubled, the total transfer cost $\Delta V$ is also multiplied by 2 . 

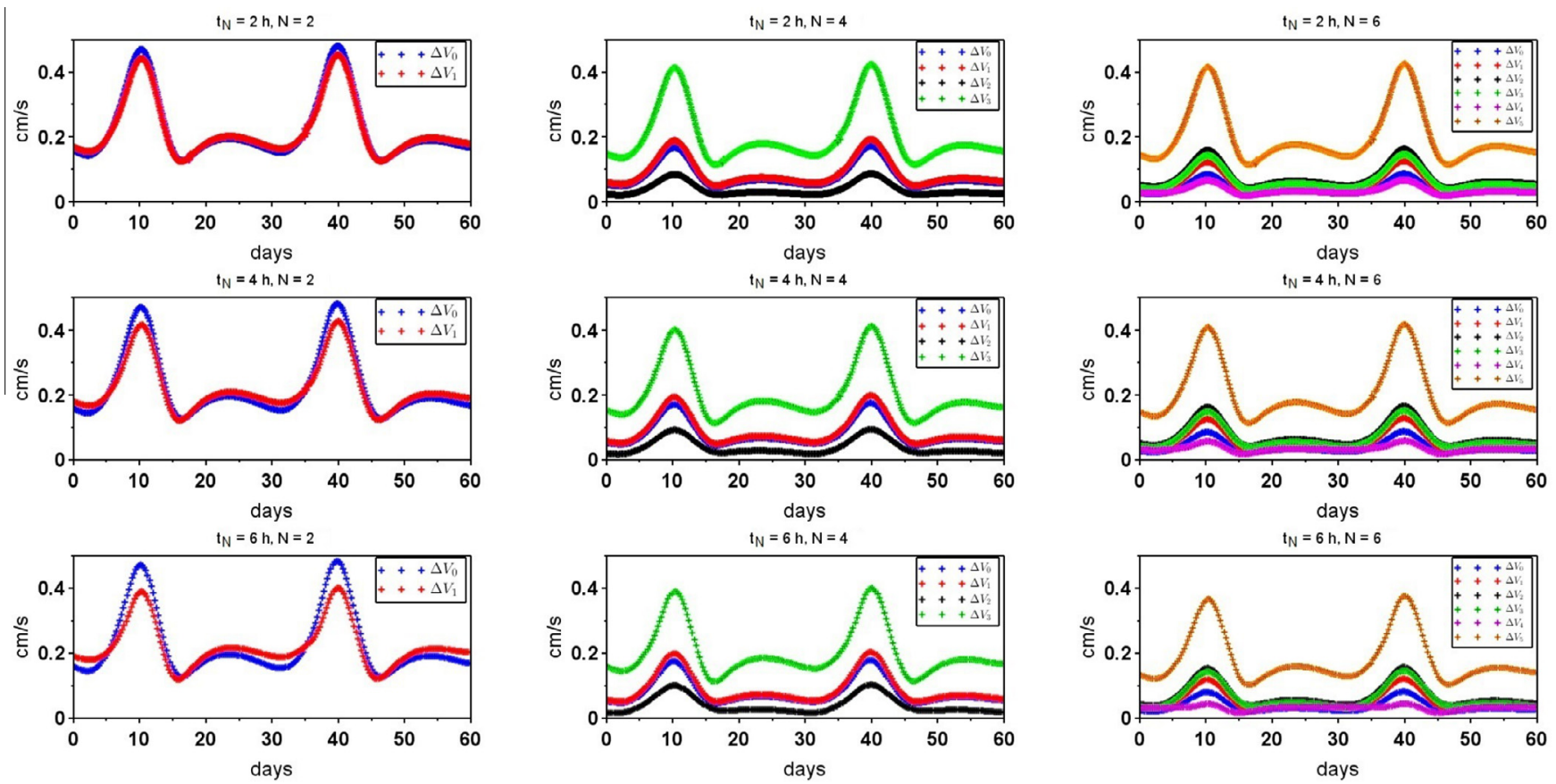

Fig. 15. Magnitude of the correction maneuvers for different sets of values of the time interval $t_{N}$ and the number of maneuvers $N \geqslant 2$, when the chief satellite moves along the reference solution QP2. The distance between the spacecraft placed initially and finally on the ZRRA cones is $2 \mathrm{~km}$, and the angle of the generatrix is equal to $25^{\circ}$. (For interpretation of the references to color in this figure legend, the reader is referred to the web version of this paper.)
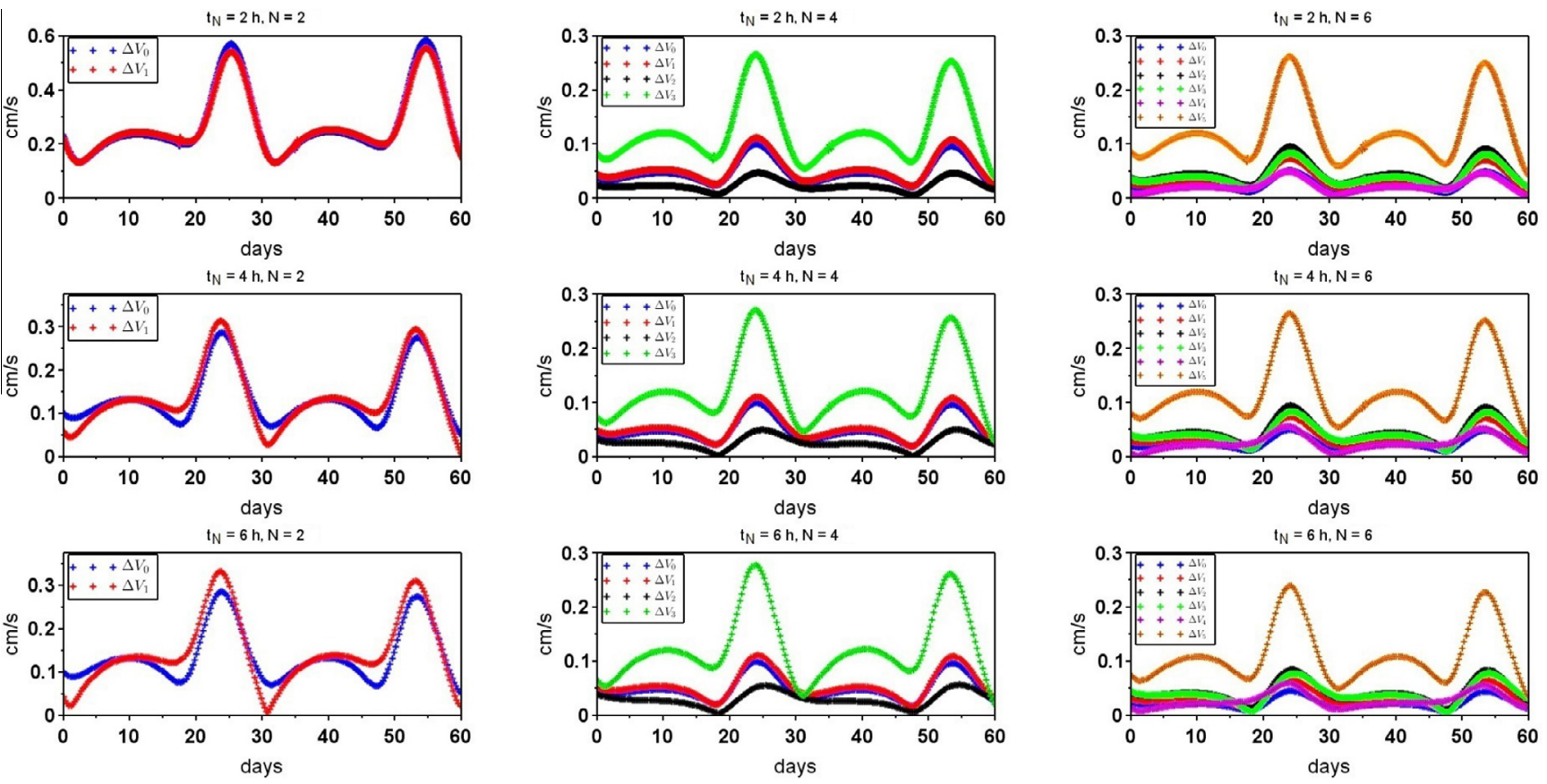

Fig. 16. Magnitude of the correction maneuvers for different sets of values of the time interval $t_{N}$ and the number of maneuvers $N \geqslant 2$, when the chief satellite moves along the reference solution QP3. The distance between the spacecraft placed initially and finally on the ZRRA cones is $2 \mathrm{~km}$, and the angle of the generatrix is equal to $25^{\circ}$. (For interpretation of the references to color in this figure legend, the reader is referred to the web version of this paper.) 


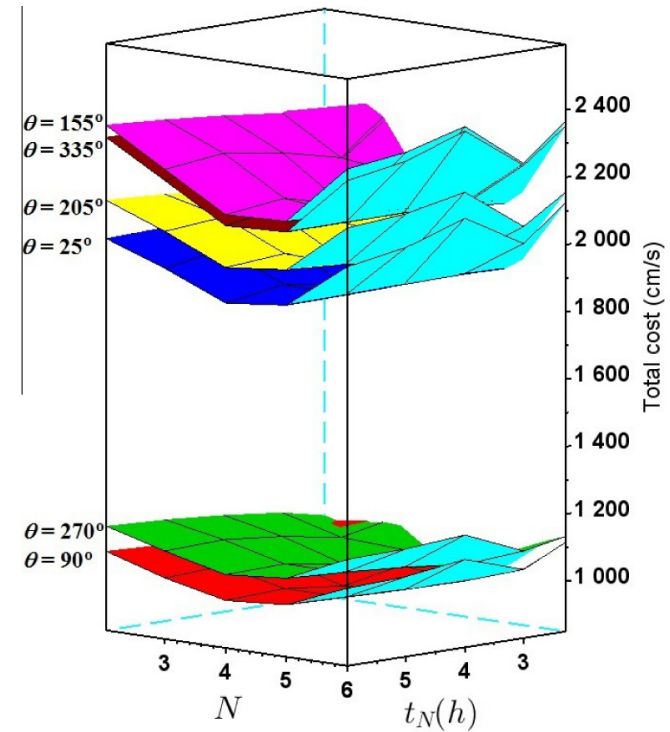

(a)

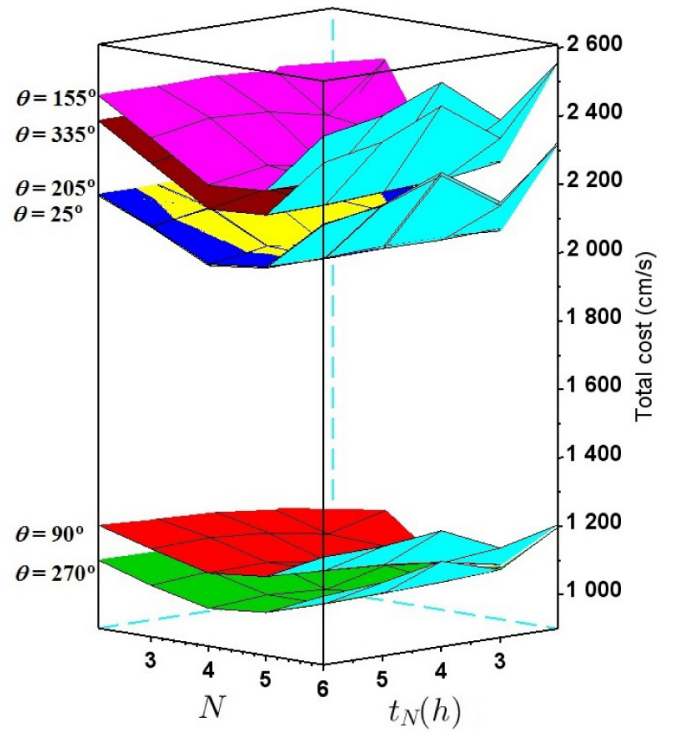

(b)

Fig. 17. Total transfer cost during 1 year for different sets of values of the angle of the generatrix $\theta\left(t_{N}\right)$, the time interval $t_{N}$ and the number of maneuvers $N \geqslant 2$, when the chief satellite moves along the reference solution QP2 (a) and QP3 (b). The distance between the spacecraft placed initially and finally on the ZRRA cones is $2 \mathrm{~km}$.

\section{Conclusions}

In this paper we have determined quadratic surfaces with zero relative radial acceleration component for the deputy vehicle in a two spacecraft formation, associated to two stable quasi-periodic trajectories suitable for formation flight about $L_{4}$ in the Sun-Earth-Moon BCFBP. Assuming a radius of a few kilometers for the geometry of the formation, the variational equation with respect to the reference trajectory allows the derivation of quadratic surfaces for the zero drift regions along each reference trajectory. Although the linear approach determines elliptic cones along the reference paths, at certain locations the cones become intersecting planes and their orientation changes.

The natural motion of the deputy spacecraft, positioned at the most favorable location with respect to the chief spacecraft, avoids large variations of the mutual distances between the deputy and chief spacecraft. This study shows that the distance of the formation with respect to the Earth affects the natural drift of the formation. In fact, the magnitude of the natural drift reaches a maximum when the formation passes close to the Earth. This affects the cost to maintain the formation on the ZRRA cones. Additionally, to avoid geometric configurations that do not satisfy the quadratic equation of the zero drift surfaces along the reference solutions, produced by the bifurcation between the elliptic cones and the intersecting planes, the generatrix in the cones is parametrized by an angle $\theta$, such that, the endpoints of the intervals for $\theta$ that satisfy the quadratic equation, can be approximated by the inclination of the intersecting planes when the bifurcation happens.
Furthermore, two control strategies were implemented to maintain the formation on the ZRRA cones considering a radius of the formation of $2 \mathrm{~km}$. The first one, the bang-off-bang control applies two control impulse maneuvers to place the deputy vehicle on the same initial or different generatrix after some interval of time. The total transfer cost of the bang-off-bang control is higher when the departure vertex is placed at the closest distance with the Earth. The best results are obtained for parallel translations, i.e. departure and arrival generatrices $\theta$ are equal, and when the formation is set at the endpoints of the intervals for $\theta$.

The second strategy carried out, applies a sequence of correction maneuvers at certain epochs, executed uniformly during a time interval required by the spacecraft to recover the formation. A set of weights is fixed, in such a way that, the weighted total $\Delta V$ consumption is minimized and the magnitude of the first maneuvers remains small. Similarly, the magnitude of the control maneuvers is higher when the formation is passing close to the Earth. Additionally, the magnitude of each maneuver decays approximately as $2^{-(N-2) / 2}$ with regard to the number of maneuvers $N \leqslant 2$. The best results are obtained when the formation is set at the locations where the previous control strategy reaches a maximum.

Now, although the BCFBP scenarios significantly affect the dynamics about $L_{4}$ point, the formation keeping $\Delta V$ requirements in this work are extremely small, on the order of $10-24 \mathrm{~m} / \mathrm{s}$ during 1 year (using the second strategy). Therefore, for small satellites with a mass of few kilograms, such small velocity changes are achievable using electric propulsion systems. 
In conclusion, this research provided a more accurate description of the relative motion about Earth-Moon triangular libration points because solar gravitational effects were modeled and added to the system. The two quasi-periodic solutions used in this work have shown to be appropriate for setting a formation about $L_{4}$. However, since BCFBP models catch the basic dynamics of formation flight about equilateral libration points in the Earth-Moon system, this analysis can be extended to more complex descriptions of relative motion at $L_{4}$ point, e.g., ephemeris-based model. New reference orbits may be included in different environments, in such a way that a more real description of the most suitable directions for formation at triangular libration points could be obtained for potential applications.

\section{Acknowledgments}

Firstly, the authors thanks the financial support of the São Paulo Research Foundation, Brazil (FAPESP), Grants 2008/06066-5, 2011/08171-3, 2011/50151-0, 2013/03233-6, and 2015/00559-3, and the Conselho Nacional para Desenvolvimento Científico e Tecnológico, Brazil (CNPq). E.E.N.M. is supported by FAPESP Grant 2011/50151-0 and CNPq. In the other hand, J. J. M. thanks the MINECO-FEDER Grant MTM2012-31714 and the Catalan Grant 2014SGR-504. G. G. thanks the MINECO-FEDER Grant MTM2013-41168-P and the Catalan Grant 2014SGR-1145.

\section{References}

Adams, J.C., Robertson, A., Zimmerman, K., et al., 1996. Technologies for spacecraft formation flying. In: Proc. of the ION GPS-96 Conference, Kansas City, MO.

Barden, B.T., Howell, K.C., 1998a. Formation flying in the vicinity of libration point orbits. Adv. Astronaut. Sci. 99 (2), 969-988.

Barden, B.T., Howell, K.C., 1998b. Fundamental motions near collinear libration points and their transitions. J. Astronaut. Sci. 46 (4), 361378.

Barden, B.T., Howell, K.C., 1999. Dynamical issues associated with relative configurations of multiple spacecraft near the Sun-Earth/ Moon L1 point. In: AAS/AIAA Astro. Specialist Conf., Girdwood, AK., AAS Paper No. 99-450.

Battrick, B., 2000. XEUS Steering Committee: X-Ray Evolving-Universe Spectroscopy: The XEUS Mission Summary. ESA Publications Division, Noordwijk, The Netherlands.

Bristow, J., Folta, D., Hartman, K.A, 2000. Formation flying technology vision. In: AIAA Space Conf., Long Beach, CA., AIAA Paper No. 2000-5194.

Burns, R., McLaughlin, C.A., Leitner, J., et al., 2000. TechSat 21: formation design, control, and simulation. In: IEEE Aero. Conf.

Cash, W., Kendrick, S., Noecker, C., et al., 2009. The new worlds observer: the astrophysics strategic mission concept study. In: Proc. SPIE, vol. 7436, article id. 743606, p. 14. http://dx.doi.org/10.1117/12 827486.

Castellà, E., Jorba, À., 2000. On the vertical families of two-dimensional tori near the triangular points of the bicircular problem. Celest. Mech. Dyn. Astron. 76 (1), 35-54. http://dx.doi.org/10.1023/ A:1008321605028.
Catlin, K.A., McLaughlin, C.A., 2007. Earth-Moon triangular libration point spacecraft formations. J. Spacecr. Rockets 44 (3), 660-670. http://dx.doi.org/10.2514/1.20152.

Defilippi, G., Jr., 1977. Station keeping at the L4 libration point: a three dimensional study (M.Sc Thesis). School of Engineering, Air Force Inst. of Tech., Wright-Patterson AFB, OH.

Farquhar, R.W., 1968. The control and use of libration-point satellites (Ph.D. Thesis). Dept. of Aeronautics and Astronautics, Stanford Univ.

Farquhar, R., 1971. the utilization of halo orbits in advanced lunar operation. NASATND-6365, GSFC, Greenbelt, Maryland.

Flick, M., 2012. Earth observing-1: preliminary technology and science validation report. Goddard Space Flight Center. <http://eol.gsfc.nasa.gov/new/validationReport/index.html $>$.

Folta, D.C., Newman, L.K., Gardner, T., 1996. Foundations of formation flying for mission to planet Earth and new millennium. In: AIAA/AAS Astro. Specialist Conf., San Diego, CA. Paper No. AIAA 96-3645.

Folta, D.C., Carpenter, J.R., Wagner, C., 2000. Formation flying with decentralized control in libration point orbits. In: Int. Symposium: Spaceflight Dynamics, Biatrritz, France.

Fridlund, C.V.M., Capaccioni, Fabrizio, 2002. Infrared space interferometry-the DARWIN mission. Adv. Space Res. 30 (9), 2135-2145. http:// dx.doi.org/10.1016/S0273-1177(02)00585-9.

Gómez, G., Lo, M., Masdemont, J.J., et al., 2001a. Simulation of formation flight near L2 for the TPF mission. In: AAS/AIAA Astro. Specialist Conf., Quebec City, Canada. Paper No. AAS 01-305.

Gómez, G., Llibre, J., Martínez, R., et al., 2001b. Dynamics and mission design near libration point orbits. Fundamentals: The Case of Triangular Libration Points, vol. II. World Scientific, Singapore.

Gómez, G., Marcote, M., Masdemont, J.J., et al., 2006. Natural configurations and controlled motions suitable for formation flying (AAS 05-347). Adv. Astronaut. Sci. 123 (2), 1513-1530.

Guinn, J., Boain, R., 1996. Spacecraft autonomous formation flying earth orbiters using GPS. In: AIAA/AAS Astro. Specialist Conf., San Diego. CA. Paper No. AIAA 96-3655.

Hamilton, N.H.. 2001. Formation flying satellite control around the L2 Sun-Earth libration point (M.S. Thesis). School of Engineering and Applied Science, George Washington Univ., Washington, DC.

Héritier, A., Howell, K., 2014. Dynamical evolution of natural formations in libration point orbits in a multi-body regime. Acta Astronaut. 102, 332-340. http://dx.doi.org/10.1016/j.actaastro.2013.10.017.

Hilbert, D., Cohn-Vossen, S., 1999. The second-order surfaces. Geom. Imagination 3, 12-19.

Hoffman, D., 1993. Station-keeping at the collinear equilibrium points of the Earth-Moon system. In: NASAJSC-26189.

Hou, X.Y., Liu, L., 2010. On quasi-periodic motions around the triangular libration points of the real Earth-Moon system. Celest. Mech. Dyn. Astron. 108 (3), 301-313. http://dx.doi.org/10.1007/ s10569-010-9305-3.

Howell, K.C., Barden, B.T., 1999. Trajectory design and stationkeeping for multiple spacecraft in formation near the Sun-Earth L1 point. In: IAF 50th International Astronautical Congress, Amsterdam, the Netherlands. Paper No. IAF/IAA 99-A707.

Howell, C., Marchand, B.G., 2005. Natural and non-natural spacecraft formations near the L1 and L2 libration points in the Sun-Earth/ Moon ephemeris system. Dyn. Syst. Int. J. 20 (1), 149-173.

How, J.P., Twiggs, R., Weidow, D., et al., 1998. Orion: a low-cost demonstration of formation flying in space using GPS. In: AIAA/AAS Astro. Specialist Conf., Boston, MA. Paper No. AIAA-98-4398.

Jorba, À., 2000. A numerical study on the existence of stable motions near the triangular points of the real Earth-Moon system. Astron. Astrophys. 364 (1), 327-338.

Jorba, À., Villanueva, J., 1997a. On the persistence of lower dimensional invariant tori under quasi-periodic perturbations. J. Nonlinear Sci. 7 (5), 427-473. http://dx.doi.org/10.1007/s003329900036.

Jorba, A., Villanueva, J., 1997b. On the normal behaviour of partially elliptic lower dimensional tori of hamiltonian systems. Nonlinearity 10 (4), 783-822. http://dx.doi.org/10.1088/0951-7715/10/4/001. 
Kapila, V., Sparks, A.G., Buffington, J.M., et al., 2000. Spacecraft formation flying: dynamics and control. J. Guidance Control Dyn. 23 (3), 561-564. http://dx.doi.org/10.2514/2.4567.

Kolenkiewicz, R., Carpenter, L., 1967. Periodic motion around the triangular libration points in the restricted problem of four bodies. Astron. J. 72 (2), 180-183.

Kolenkiewicz, R., Carpenter, L., 1968. Stable periodic orbits about the Sun perturbed Earth-Moon triangular points. AIAA J. 6 (7), 13011304.

Marchand, G., Howell, K.C., 2005. Control strategies for formation flight in the vicinity of the libration points. J. Guidance Control Dyn. 28 (6), $1210-1219$

Munoz, J.P., 2008. Sun-perturbed dynamics of a particle in the vicinity of the Earth-Moon triangular libration points (Ph.D. Thesis). Dept. of Aerospace Engineering, University of Texas at Austin.

O'Neill, G.K., 1974. The colonization of space. Phys. Today 27 (9), 32-40. http://dx.doi.org/10.1063/1.3128863.

Perea, L., Elosegui, P., Gómez, G., 2009. Extension of the cucker-smale control law to space flight formations. J. Guidance Control Dyn. 32 (2), 527-537. http://dx.doi.org/10.2514/1.36269.

Press, W.H., Teukolsky, S.A., Vetterling, W.T., et al., 1992. Numerical Recipes in C: The Art of Scientic Computing, second ed. Cambridge University Press, Cambridge.

Sabol, C., Burns, R., McLaughlin, C.A., 2001. Satellite formation flying design and evolution. J. Spacecr. Rockets 38 (2), 270-278. http:// dx.doi.org/10.2514/2.3681.

Salazar, F., Masdemont, J.J., Gómez, G., et al., 2014. Zero, minimum and maximum relative radial acceleration for planar formation flight dynamics near triangular libration points in the Earth-Moon system. Adv. Space Res. 54 (9), 1838-1857. http://dx.doi.org/10.1016/ j.asr.2014.07.018.
Salazar, F., Winter, O.C., Macau, E.E., et al., 2015. Natural formations at the Earth-Moon triangular point in perturbed restricted problems. Adv. Space Res. 56 (1), 144-162. http://dx.doi.org/10.1016/ j.asr.2015.03.028.

Schutz, B.E., 1977. Orbital mechanics of space colonies at 14 and 15 of the Earth-Moon system. In: AIAA Astro. Specialist Conf., Los Angeles, CA. AIAA Paper No. 77-33.

Sholomitsky, G.B., Prilutsky, O.F., Rodin, V.G., 1977. Infra-red space interferometer. In: 28th Int. Astro. Fed. Congress, Praha, Czechoslovakia. Paper IAF-77-68.

Simó, C., Martínez, R., Gómez, G., et al., 1987. Relative motion near the triangular libration points in the Earth-Moon system. In: ESA Workshop on Optical Interferometry in Space, Granada, Spain. ESA SP-273.

Simó, C., Gómez, G., Jorba, À., et al., 1995. The bicircular model near the triangular libration points of the RTBP. In: From Newton to Chaos, pp. 343-370.

Stoer, J., Bulirsch, R., 1980. Introduction to Numerical Analysis, 1st ed. Springer Verlag, New York, NY.

Tapley, B.D., Lewallen, J.M., 1964. Solar influence on satellite motion near the stable Earth-Moon libration points. AIAA J. 2 (4), 728-732.

Ticker, R.L., Azzolini, J.D., 2000. 2000 Survey of distributed spacecraft technologies and architectures for NASA's Earth science enterprise in the 2010-2015 timeframe. NASA/TM-2000-209964.

Wolaver, L.E., 1965. Effect of initial configurations in libration point motion. In: AIAA/ION Astro. Specialist Conf., Monterey, California. AIAA Paper No. 65-684.

Wong, F.Y.W., 2009. Formation-keeping strategies at the Earth-Moon L4 triangular libration point (M.Sc. Thesis). Department of Aerospace Engineering, Ryerson Univ., Toronto, Ontario, Canada. 